\title{
SOLVING LP PROBLEMS VIA WEIGHTED CENTERS
}

\author{
AIPING LIAO* AND MICHAEL J. TODD ${ }^{\dagger}$
}

July 12, 1993

\begin{abstract}
The feasibility problem for a system of linear inequalities can be converted into an unconstrained optimization problem using ideas from the ellipsoid method, which can be viewed as a very simple minimization technique for the resulting nonlinear function. Using more sophisticated algorithms, we develop and investigate more efficient methods, which lead to two kinds of weighted centers for the feasible set. With these centers, we develop new algorithms for solving linear programming problems.
\end{abstract}

Key words. weighted center, the ellipsoid method, Newton's method, linear programming.

AMS subject classifications. $65 \mathrm{~K}, 90 \mathrm{C}$

1. Introduction and history of centers. In this paper we will consider linear programming problems with the following form:

$$
\begin{aligned}
& \min c^{T} x \\
& l \leq A^{T} x \leq u
\end{aligned}
$$

where $A$ is an $n \times m$ matrix with full rank. We assume that $m \geq n \geq 2$ and $l<u$. As we know, any linear programming problems can be reduced to the so-called inequality form

$$
\begin{aligned}
& \min _{\tilde{A}^{T} x \leq b} \tilde{c}^{T} x \\
& x \geq 0
\end{aligned}
$$

If the input data are all integers, (ILP) is equivalent to

$$
\begin{aligned}
& \min \tilde{c}^{T} x \\
& \tilde{A}^{T} x \leq b \\
& (l=) 0 \leq x \leq u
\end{aligned}
$$

where $u=2^{L} e$ with $L$ the input length of (ILP), which can be easily adapted to a form of (LP) (see, e.g., Burrell and Todd [5]). So, theoretically speaking, our form (LP) is not restrictive. On the other hand, since "many linear programming problems involve explicit upper bounds on individual variables [in the standard form]" (Chvátal [6]) - especially, the upper bounds may stand for limits on resources - we thus often, in practice, are confronted with linear programming problems with the following form:

$$
\begin{aligned}
& \min c^{T} x \\
& A^{T} x \leq b \\
& (l=) 0 \leq x \leq u
\end{aligned}
$$

* Advanced Computing Research Institute and Center for Applied Mathematics, Cornell University, Ithaca, New York 14853, partially supported by the Cornell Theory Center, which receives major funding from the National Science Foundation and IBM Corporation, with additional support from the State of New York and its Corporate Reaserch Institutes; and by NSF, AFOSR, and ONR through grant DMS-8920550.

$\dagger$ School of Operations Research and Industrial Engineering, Cornell University, Ithaca, New York 14853, partially supported by NSF, AFOSR, and ONR through grant DMS-8920550. 
which, again, can be converted to our form (LP). Thus (LP) is not very restrictive in practice either. In Section 2 and 3 we will discuss methods for solving the linear system

$$
l \leq A^{T} x \leq u
$$

and introduce two weighted centers and then, in Section 4, we develop algorithms for solving (LP) via these two kinds of center. We also give encouraging numerical results in Section 3 and 4. Section 5 presents a summary and conclusion.

One recent development in linear programming can be regarded as a study of various concepts of "center". A center of a polytope $P \subset R^{n}$ must be in the (relative) interior of $P$. Since 1984 when Karmarkar proposed his famous projective algorithm, interior point methods have become the mainstream of research in linear programming. Basically speaking, in this field, there are two aspects are under study. One is the "center" which provides the interior point; the other is the way to move the center and the merit function for measuring the progress made by this movement.

The first kind of "center" that was used in optimization is the center of gravity or centroid used by Levin [16] in his algorithm, the method of central sections, for minimization of a convex function over a convex polytope $P$ (see also Newman [21]). In that paper the centroid is used as the test point. If the current centroid, say $x^{k}$, satisfies the convergence criterion, stop with a satisfactory approximate solution; otherwise, cut the current polytope $P^{k}$ into two pieces by a hyperplane through $x^{k}$, find out which part the optimum lies in, and then throw away the other part. The volume of the polytope under consideration is thus reduced, and this procedure can be repeated until a satisfactory centroid is obtained. This method is very concise and $1-\exp (-1)$ is a guaranteed reduction of the volume of successive polytopes. The disadvantage is the difficulty of calculating the centroid.

Yudin and Nemirovskii [31] discuss the computational difficulties of Levin's method and propose a modified method of centered cross-sections, using ellipsoids instead of polyhedra. This modified method is computationally implementable. They also point out that this ellipsoid method is a special case of Shor's algorithm [24] with space dilation in the direction of the subgradient. Shor [25] independently developed the ellipsoid method. Later, in 1979, Khachiyan [14] showed that the ellipsoid method is a polynomial time algorithm for solving linear programming problems.

We note that John's result [13] shows that for every convex polytope $P \subset R^{n}$, the minimum volume ellipsoid containing $P$ exists and is unique. It is easy to show that the center of this minimum volume ellipsoid is in $P$, for otherwise, by one step of the ellipsoid algorithm a smaller ellipsoid containing $P$ can be obtained. Thus, the center of this ellipsoid can be used as a "center". However, the smallest ellipsoid containing $P$ is hard to find in general, and so is its center.

Tarasov, Khachiyan and Erlich [27] study the method of inscribed ellipsoids. Actually, the center of the maximal inscribed ellipsoid for a polytope can be regarded as a "center". In [15], Khachiyan and Todd discuss the problem of approximating the maximal inscribed ellipsoid and related problems. They also propose algorithms for finding these ellipsoids.

Renegar [22] uses the "analytical center" (see also Sonnevend [26]) to develop his algorithm for linear programming problems, which is polynomial time bounded. The analytical center is easier to approximate compared with the centroid. Actually, in his algorithm, Renegar showed that an " $\epsilon$-analytical center" is enough. Unlike the centroid, the analytical center is not analytically independent; it depends on the way 
in which the polytope $P$ is represented. Renegar [22] makes use of this property to improve the convergence of his algorithm by adding some extra constraints.

In the paper [30] Vaidya introduces the "volumetric center" which is the center of the ellipsoid with largest volume among a certain set of ellipsoids that are contained in $P$ and proposes an algorithm with a better global convergence rate and time complexity than the ellipsoid method.

The "centers" of $P$ we propose here are the centers of the ellipsoids with smallest volumes among certain sets of ellipsoids that contain $P$. These ellipsoids are of simpler structure and are relatively easier to construct.

2. Model I and the associated center. We consider the feasibility problem:

$$
\text { find } \bar{x} \text { such that } \bar{x} \in P:=\left\{x: l \leq A^{T} x \leq u\right\} .
$$

For convenience we denote $r:=\frac{l+u}{2}$ and $s:=\frac{u-l}{2}$. We also denote by $e$ the all-one vector and $e_{j}$ the $j$-th column of the identity matrix. In [5] Burrell and Todd proposed a parallel-cut ellipsoid algorithm based on the results of Todd [29].

Note that $P$ can be alternately written as

$$
P=\left\{x \in R^{n}:\left(a_{i}^{T} x-l_{i}\right)\left(a_{i}^{T} x-u_{i}\right) \leq 0, i=1, \ldots, m\right\}
$$

where $a_{i}$ is the $i$-th column of $A$ and $l_{i}, u_{i}$ are the corresponding components of $l, u$ respectively. Now choose a nonnegative diagonal matrix $D=\operatorname{diag}(d)=\operatorname{diag}\left(d_{1}, \ldots, d_{m}\right)$, and combine the inequalities above with weights $d_{i}$. We thus obtain a set

$$
E:=E(d):=\left\{x \in R^{n}:\left(A^{T} x-l\right)^{T} D\left(A^{T} x-u\right) \leq 0\right\} .
$$

It is obvious that $P \subset E$. We suppose that $A D A^{T}$ is nonsingular. Then $E$ is actually an ellipsoid. Further calculation shows that

$$
E=\left\{x \in R^{n}:\left(x-x_{c}\right)^{T} A D A^{T}\left(x-x_{c}\right) \leq x_{c}^{T}\left(A D A^{T}\right) x_{c}-l^{T} D u\right\}
$$

where

$$
x_{c}:=x_{c}(d):=\left(A D A^{T}\right)^{-1} A D r
$$

is the center of $E$.

If the current center violates some constraint, say, $l_{i} \leq a_{i}^{T} x \leq u_{i}$, by the results of Todd [29] we can construct a new ellipsoid that contains that part of the previous one between the parallel hyperplanes $a_{i}^{T} x=l_{i}$ and $a_{i}^{T} x=u_{i}$, and the volume of the ellipsoid decreases by a factor which is, at worst, $\exp \left(-\frac{1}{2(n+1)}\right)$.

Consider the problem

$$
\begin{aligned}
& \min v(d):=f(d) \cdot h(d) \quad\left(\mathrm{P}_{f h}\right) \\
& \text { s.t. } d \in \mathcal{D},
\end{aligned}
$$

where

$$
\begin{aligned}
f(d) & :=r^{T} D A^{T}\left(A D A^{T}\right)^{-1} A D r-l^{T} D u=x_{c}^{T} A D A^{T} x_{c}-l^{T} D u, \\
h(d) & :=\left(\operatorname{det}\left(A D A^{T}\right)\right)^{-\frac{1}{n}}, \text { and } \\
\mathcal{D} & :=\left\{d: d \geq 0, A D A^{T} \text { is nonsingular }\right\} .
\end{aligned}
$$

Note that the volume of $E(d)$ is $\kappa_{n} \cdot(v(d))^{\frac{n}{2}}$, where $\kappa_{n}$ is the volume of the unit ball in $R^{n}$. Liao and Todd [18] show that the Burrell-Todd algorithm is basically 
the coordinate descent method applied to $\left(\mathrm{P}_{f h}\right)$, together with rules for updating the bounds $l$ and $u$. They also propose a simpler way to perform the updating.

As we know Newton's method is fast in practice while the coordinate descent algorithm is usually considered not fast enough for practical use. But here the function $v(d)$ is a homogeneous function of degree 0 , and it can be shown (see, for example, Liao and Todd [18]) that

$$
\begin{aligned}
\nabla v(d)+\nabla^{2} v(d)^{T} d & =0, \\
d^{T} \nabla v(d) & =0
\end{aligned}
$$

so $d$ is the Newton direction, as well as a direction of constancy for the function, and therefore useless. The other difficulty preventing us from using Newton's method is that we do not even know if there exists an optimal solution to problem $\left(\mathrm{P}_{f h}\right)$. We thus try to modify our model so that Newton's method can be applied.

The first new model is as follows.

$$
\begin{aligned}
& \min F(d):=f(d)+h(d) \quad\left(\mathrm{P}_{f+h}\right) \\
& \text { s. t. } d \in \mathcal{D} .
\end{aligned}
$$

We note that if $f(d)>0$, by the arithmetic-geometric mean inequality, $F(d) \geq$ $2 \sqrt{v(d)}$. Moreover, it is easy to see that $f$ is homogeneous of degree 1 and $h$ of degree -1 ; thus, for any given $d \in \mathcal{D}, F(d)$ attains its minimum over the half-line $\{\lambda d: \lambda \in R, \lambda>0\}$ at $d^{*}:=\sqrt{\frac{h(d)}{f(d)}} \cdot d$ which gives the value $F\left(d^{*}\right)=2 \sqrt{v\left(d^{*}\right)}$.

2.1. Properties of $\left(\mathrm{P}_{f+h}\right)$. In the following we give some properties of $\left(\mathrm{P}_{f+h}\right)$. Proposition 2.1. $\mathcal{D}$ defined above is a convex set.

Proof. We note that for any $d \in \mathcal{D}, A D A^{T}$ is positive definite. Suppose we have $d^{1}, d^{2} \in \mathcal{D}$. Then both $A D^{1} A^{T}$ and $A D^{2} A^{T}$ are positive definite. For any $\lambda$ such that $0 \leq \lambda \leq 1$, let $d=\lambda d^{1}+(1-\lambda) d^{2}$, and we have $A D A^{T}=\lambda A D^{1} A^{T}+(1-\lambda) A D^{2} A^{T}$ which is also positive definite and thus nonsingular. Since $d^{1} \geq 0, d^{2} \geq 0$ imply $d \geq 0$, we have $d \in \mathcal{D}$, which in turn shows that $\mathcal{D}$ is a convex set.

Now we show that both $f(d)$ and $h(d)$ are convex functions over $\mathcal{D}$. We first prove some lemmas.

Lemma 2.2 .

$$
\begin{aligned}
& \frac{\partial\left(A D A^{T}\right)^{-1}}{\partial d_{i}}=-\left(A D A^{T}\right)^{-1} a_{i} a_{i}^{T}\left(A D A^{T}\right)^{-1}, \\
& \frac{\partial\left(\operatorname{det}\left(A D A^{T}\right)\right)}{\partial d_{i}}=\operatorname{det}\left(A D A^{T}\right) a_{i}^{T}\left(A D A^{T}\right)^{-1} a_{i} .
\end{aligned}
$$

Proof. The proof is easy using the rank-1 update formulae. $\square$

Lemma 2.3. For $i=1, \ldots, m$, the $i-$ th components of $\nabla f(d)$ and $\nabla h(d)$ are:

$$
\begin{aligned}
& \frac{\partial f(d)}{\partial d_{i}}=-\left(a_{i}^{T} x_{c}-l_{i}\right)\left(a_{i}^{T} x_{c}-u_{i}\right), \\
& \frac{\partial h(d)}{\partial d_{i}}=-\frac{1}{n} \operatorname{det}\left(A D A^{T}\right)^{-\frac{1}{n}} a_{i}^{T}\left(A D A^{T}\right)^{-1} a_{i} .
\end{aligned}
$$

Proof. It can be obtained from direct calculations using Lemma 2.2.

If $B=\left(b_{i j}\right)_{m \times n}, C=\left(c_{i j}\right)_{m \times n}$ are two $m$ by $n$ matrices, the "Schur Product" of $B$ and $C$ is defined by $B \circ C:=\left(b_{i j} c_{i j}\right)_{m \times n}$. The following result can be found, for example, in [12]. 
Lemma 2.4. If $B$ and $C$ are $m$ by $m$ positive semidefinite matrices, so is $B \circ C$. Proposition 2.5. $f$ and $h$ are convex functions over $\mathcal{D}$, and hence so is $F=$ $f+h$.

Proof. By using Lemma 2.2, direct calculations give the Hessian of $f$ as follows.

$$
H_{f}=2 \cdot \operatorname{diag}\left(A^{T} x_{c}-r\right) A^{T}\left(A D A^{T}\right)^{-1} A \cdot \operatorname{diag}\left(A^{T} x_{c}-r\right)
$$

which is obviously positive semidefinite for all $d \in \mathcal{D}$. As for $h$, calculations using Lemma 2.2 give us the following expression for the $(i, j)$-element of the Hessian of $h$ :

$H_{h}(i, j)=\frac{1}{n^{2}} \operatorname{det}\left(A D A^{T}\right)^{-\frac{1}{n}}\left[a_{i}^{T}\left(A D A^{T}\right)^{-1} a_{i} \cdot a_{j}^{T}\left(A D A^{T}\right)^{-1} a_{j}+n\left(a_{i}^{T}\left(A D A^{T}\right)^{-1} a_{j}\right)^{2}\right]$.

Let $\nu_{i}:=\left(A D A^{T}\right)^{-\frac{1}{2}} a_{i}$. Then

$$
H_{h}(i, j)=\frac{1}{n^{2}} \operatorname{det}\left(A D A^{T}\right)^{-\frac{1}{n}}\left[\left(\nu_{i}^{T} \nu_{i}\right) \cdot\left(\nu_{j}^{T} \nu_{j}\right)+n\left(\nu_{i}^{T} \nu_{j}\right)^{2}\right]
$$

which shows that $H_{h}$ is positive semidefinite, since the first term in the square brackets is a matrix of a product of a vector by its transpose, which is thus positive semidefinite, while the second term is positive semidefinite from Lemma 2.4. $\square$

Lemma 2.6. If $\operatorname{int}(P) \neq \emptyset$, then

$$
f(d)>0, \text { for all } d \in \mathcal{D} .
$$

Proof. For $d \in \mathcal{D}, E(d)$ is an ellipsoid that contains $P$. If $\operatorname{int}(P)$ is not empty, $E(d)$ is a full dimensional ellipsoid; thus the volume of $E(d)$ is positive, i.e. $v(d)>0$. Therefore $f(d) h(d)>0$, and so $f(d)>0$, since $h(d)>0$. $\square$

In the following we show that $f$ can be used to determine whether $P=\emptyset$. This result provides us a tool that is useful later in the analysis of the output of our model.

Theorem 2.7. If $l<u$, and $A$ is of full rank, then

$$
P=\emptyset \Longleftrightarrow f(d)<0 \text {, for some } d \in \mathcal{D} .
$$

Proof. " $\Longrightarrow "$. First of all, we recall Helly's Theorem which can be found, e.g., in Rockafellar [23].

Let $\left\{C_{i} \mid i \in \mathcal{I}\right\}$ be a finite collection of convex sets in $R^{n}$. If every subcollection consisting of $n+1$ or fewer sets has a non-empty intersection, then the entire collection has a non-empty intersection.

If $P=\emptyset$, by Helly's Theorem there are $n+1$ constraints which are inconsistent. If the rank of the set of the corresponding $a_{i}$ 's is $k<n$, then, since Helly's Theorem is invariant under affine transformation, we can find $k+1$ constraints which are inconsistent too, and since we assume that $A$ is of full rank, we can expand these $k+1$ ones to $n+1$ constraints which are inconsistent and the corresponding $a_{i}$ 's are of rank $n$.

Therefore, if $P=\emptyset$, there are $n+1$ constraints, say the first $n+1$ ones, which are inconsistent and the corresponding $a_{i}$ 's are of rank $n$. Without loss of generality, we assume that

$$
\bar{A}=[I, a], \text { and } l_{i}=-1, u_{i}=1, \forall i=1, \ldots, n, \text { and }\|a\|_{1}=1,
$$

and the corresponding system

$$
\left(\begin{array}{c}
-e \\
l_{n+1}
\end{array}\right) \leq\left(\begin{array}{c}
I \\
a^{T}
\end{array}\right) x \leq\left(\begin{array}{l}
e \\
u_{n+1}
\end{array}\right)
$$


is inconsistent. We claim that there exists $d \in\left\{d \in \mathcal{D}: d_{i}=0, \forall i=n+2, \ldots, m\right\}$ for which $f(d)<0$. Equivalently, we show that the claim is true for system (10). We denote $s:=\frac{u-l}{2}$ as before.

$$
\begin{aligned}
f(d)= & r^{T} D \bar{A}^{T}\left(\bar{A} D \bar{A}^{T}\right)^{-1} \bar{A} D r-l^{T} D u \\
= & \left(0, \ldots, 0, d_{n+1} r_{n+1}\right) \bar{A}^{T}\left(D^{\prime-1}-\frac{d_{n+1} D^{\prime-1} a a^{T} D^{\prime-1}}{1+d_{n+1} a^{T} D^{\prime-1} a}\right) \bar{A}\left(\begin{array}{l}
0 \\
\vdots \\
0 \\
d_{n+1} r_{n+1}
\end{array}\right) \\
& +e^{T} d^{\prime}-d_{n+1} l_{n+1} u_{n+1} \\
& \left(\text { where } d^{\prime}=\left(d_{1}, \ldots, d_{n}\right)^{T} \in R^{n}\right) \\
= & \frac{d_{n+1}^{2} r_{n+1}^{2} a^{T} D^{\prime-1} a}{1+d_{n+1} a^{T} D^{\prime-1} a}+e^{T} d^{\prime}-d_{n+1} l_{n+1} u_{n+1} \\
= & \left(1+d_{n+1} a^{T} D^{\prime-1} a\right)^{-1}\left\{d_{n+1}^{2} r_{n+1}^{2} a^{T} D^{\prime-1} a+e^{T} d^{\prime}+d_{n+1} e^{T} d^{\prime} a^{T} D^{\prime-1} a\right. \\
& \left.-d_{n+1} l_{n+1} u_{n+1}-d_{n+1}^{2} l_{n+1} u_{n+1} a^{T} D^{\prime-1} a\right\} \\
= & \left(1+d_{n+1} a^{T} D^{\prime-1} a\right)^{-1}\left\{d_{n+1}^{2} s_{n+1}^{2} a^{T} D^{\prime-1} a+e^{T} d^{\prime}+d_{n+1} e^{T} d^{\prime} a^{T} D^{\prime-1} a\right. \\
& \left.-d_{n+1} l_{n+1} u_{n+1}\right\} \\
= & \left(1+d_{n+1} a^{T} D^{\prime-1} a\right)^{-1}\left\{q\left(d_{n+1}\right)\right\}
\end{aligned}
$$

where $q\left(d_{n+1}\right)$ is a quadratic function of $d_{n+1} \cdot q\left(d_{n+1}\right)$ is minimized by

$$
d_{n+1}=\frac{l_{n+1} u_{n+1}-e^{T} d^{\prime} \sum_{i=1}^{n} \frac{a_{i}^{2}}{d_{i}}}{2 s_{n+1}^{2} \sum_{i=1}^{n} \frac{a_{i}^{2}}{d_{i}}}
$$

where $a=\left(a_{1}, \ldots, a_{n}\right)^{T}$. Plugging this $d_{n+1}$ into the above formula for $f(d)$, we have

$$
\begin{aligned}
f(d)= & \left(1+d_{n+1} a^{T} D^{\prime-1} a\right)^{-1}\left(4 s_{n+1}^{2} \sum_{i=1}^{n} \frac{a_{i}^{2}}{d_{i}}\right)^{-1}\left\{-\left(l_{n+1} u_{n+1}-e^{T} d^{\prime} \sum_{i=1}^{n} \frac{a_{i}^{2}}{d_{i}}\right)^{2}\right. \\
& \left.+4 s_{n+1}^{2} e^{T} d^{\prime} \sum_{i=1}^{n} \frac{a_{i}^{2}}{d_{i}}\right\} \\
= & :\left(1+d_{n+1} a^{T} D^{\prime-1} a\right)^{-1}\left(4 s_{n+1}^{2} \sum_{i=1}^{n} \frac{a_{i}^{2}}{d_{i}}\right)^{-1}\left\{\mathcal{L}\left(d_{1}, \ldots, d_{n}\right)\right\} .
\end{aligned}
$$

We now show that there is $d^{\prime}=\left(d_{1}, \ldots, d_{n}\right)$ such that $\mathcal{L}\left(d_{1}, \ldots, d_{n}\right)<0$. By scaling $d^{\prime}$ we can assume $e^{T} d^{\prime}=1$, and

$$
\mathcal{L}\left(d^{\prime}\right)<0 \Longleftrightarrow\left|l_{n+1} u_{n+1}-\sum_{i=1}^{n} \frac{a_{i}^{2}}{d_{i}}\right|>2 s_{n+1} \sqrt{\sum_{i=1}^{n} \frac{a_{i}^{2}}{d_{i}}} .
$$

Since system (10) has no solution, thus, if we let

$$
\mathcal{X}:=\left\{x \in R^{n}:-1 \leq x_{i} \leq 1, i=1, \ldots, n\right\},
$$

then either $a^{T} x<l_{n+1}, \forall x \in \mathcal{X}$, or $a^{T} x>u_{n+1}, \forall x \in \mathcal{X}$. We assume the first case.

On the other hand, $a^{T} x$ is maximized over $\mathcal{X}$ by some vertex of $\mathcal{X}$, which gives an optimal value as $\sum_{i=1}^{n}\left|a_{i}\right|=\|a\|_{1}=1$. Therefore, $l_{n+1}>1$. 
(i) If $a_{i} \neq 0, \forall i=1, \ldots, n$, by taking $d_{i}=\left|a_{i}\right|$, and noting that $l_{n+1}>1$, we thus have

$$
l_{n+1}^{2}+2 s_{n+1} l_{n+1}-\sum_{i=1}^{n} \frac{a_{i}^{2}}{d_{i}}=l_{n+1}^{2}-1+2 s_{n+1} l_{n+1}>2 s_{n+1} \cdot 1=2 s_{n+1} \sqrt{\sum_{i=1}^{n} \frac{a_{i}^{2}}{d_{i}}}
$$

so that (11) holds.

(ii) If some $a_{i}{ }^{\prime} s$ are zeros, then we take

$$
d_{i}= \begin{cases}\left|a_{i}\right|-\epsilon & \text { if } a_{i} \neq 0 \\ \frac{n^{\prime}}{n-n^{\prime}} \epsilon & \text { if } a_{i}=0\end{cases}
$$

where $n^{\prime}$ is the number of $a_{i}$ with $a_{i} \neq 0$. By letting $\epsilon$ be very small we can obtain $d_{1}, \ldots, d_{n}$ such that (11) holds.

Thus we have proved that if $P=\emptyset$, there exists $d$ such that $f(d)<0$.

"£". If there is $d$ such that $f(d)<0$, and $P \neq \emptyset$, then, by taking $\bar{l}_{i}=l_{i}-\delta, \bar{u}_{i}=$ $u_{i}+\delta$, and noting that $f$ is a continuous function of $l$, $u$, for any given $d$, we can choose small $\delta$ so that for the corresponding system we have

$$
\operatorname{int}(P) \neq \emptyset, f(d)<0
$$

which contradicts Lemma 2.6 .

We now make the assumption:

(A1): $\operatorname{int}(P) \neq \emptyset$ and $\left\|a_{i}\right\| \neq 0$, for all $i=1, \ldots, m$.

Here and throughout we denote by $\|\cdot\|$ the $l_{2}$ norm.

Theoretically speaking, if $P$ is not empty then by a perturbation we can get an equivalent system which satisfies $\operatorname{int}(P) \neq \emptyset$. Thus (A1) is not that restrictive.

TheOREm 2.8. Suppose (A1) holds. Then $\left(\mathrm{P}_{f+h}\right)$ is a convex programming problem, and the solution set, denoted $\mathcal{S}_{f+h}$, is not empty.

Proof. From the previous lemmas, $\left(\mathrm{P}_{f+h}\right)$ is a convex program. We now prove its solution set is not empty. Define

$$
\tilde{F}(d):= \begin{cases}f(d)+h(d) & \text { if } d \in \mathcal{D} \\ +\infty & \text { otherwise. }\end{cases}
$$

Since $\tilde{F}$ is not identically $+\infty$, and $\tilde{F}(d)>-\infty$ for every $d, \tilde{F}$ is a proper convex function.

On the other hand, since $\tilde{F}(d)$ is continuous on $\operatorname{dom}(\tilde{F})=\mathcal{D}$, if $d_{k}$ lies in the relative interior of $\mathcal{D}$ and $d_{k} \rightarrow d$, then, by Lemma 2.6 ,

$$
\tilde{F}\left(d_{k}\right) \longrightarrow\left\{\begin{array}{ll}
F(d) & \text { if } d \in \mathcal{D} \\
+\infty & \text { if } d \notin \mathcal{D}
\end{array}\right\}=\tilde{F}(d)
$$

So $\tilde{F}$ is closed (since $c l \tilde{F}=\tilde{F}$ ).

In the following we prove that $\tilde{F}$ has no direction of recession in $R^{m}$. Suppose we are given a direction, say $y$. We show that $y$ cannot be a recession direction of $\tilde{F}$. Since $\tilde{F}(d)=\infty$ for $d \notin R_{+}^{m}$ where $R_{+}^{m}:=\left\{d \in R^{m}: d \geq 0\right\}$, no $y \notin R_{+}^{m}$ can be a recession direction for $\tilde{F}$. Therefore we need only consider $y \in R_{+}^{m}$.

Suppose we are given $y \in R_{+}^{m}$ and $y \neq 0$. Without loss of generality we assume $y_{1} \neq 0$. Since $A$ is of full rank and $a_{1} \neq 0$, there is a set of $n$ columns of $A$ including $a_{1}$ which is linearly independent; and by Cauchy's formula,

$$
h(e+\lambda y) \leq \alpha^{-\frac{2}{n}}\left(1+\lambda y_{1}\right)^{-\frac{1}{n}}
$$


where $\alpha$ is the determinant of the matrix formed by the $n$ independent columns. Thus $h(e+\lambda y) \longrightarrow 0$. On the other hand, since $\operatorname{int}(P)$ is not empty and $P \subset E$, therefore there is $\delta>0$ such that

$$
f(e+\lambda y) h(e+\lambda y)>\delta>0 .
$$

Thus

$$
f(e+\lambda y) \longrightarrow \infty \text { as } \lambda \longrightarrow \infty,
$$

which shows $f$, thus $\tilde{F}$, has no recession direction in $R_{+}^{m}$.

We have thus proved that $\tilde{F}$ has no recession direction in $R^{m}$. Therefore, by Theorem 27.3 of Rockafellar [23], $\tilde{F}$ attains its minimum in $R^{m}$. Equivalently, $F$ attains its minimum over $\mathcal{D}$, i.e., $\mathcal{S}_{f+h}$ is not empty. $\square$

The following corollaries show that, under assumption $(\mathrm{A} 1),\left(\mathrm{P}_{f+h}\right)$ and $\left(\mathrm{P}_{f h}\right)$ are essentially the same.

CoRollary 2.9. Under (A1), the solution set of

$$
\begin{array}{ll}
\min v:=f \cdot h & \left(\mathrm{P}_{f h}\right) \\
\text { s.t. } d \in \mathcal{D}
\end{array}
$$

is not empty.

Proof. Suppose $d^{*}$ is an optimal solution to $\left(\mathrm{P}_{f+h}\right)$. We show that $d^{*}$ is an optimal solution to $\left(\mathrm{P}_{f h}\right)$. Otherwise, there exists $\bar{d}$ such that $v\left(d^{*}\right)>v(\bar{d})$. Since $f(d)>0$ for $d \in \mathcal{D}$, so we can let $\lambda=\sqrt{\frac{h(\bar{d})}{f(d)}}$, and have

$$
2 \sqrt{v(\bar{d})}=2 \sqrt{v(\lambda \bar{d})}=f(\lambda \bar{d})+h(\lambda \bar{d})
$$

which leads to

$$
f\left(d^{*}\right)+h\left(d^{*}\right) \geq 2 \sqrt{v\left(d^{*}\right)}>2 \sqrt{v(\bar{d})}=2 \sqrt{v(\lambda \bar{d})}=f(\lambda \bar{d})+h(\lambda \bar{d}),
$$

a contradiction.

As a matter of fact, if we let $\mathcal{S}_{f h}, \mathcal{S}_{f+h}$ be the solution sets of $\left(\mathrm{P}_{f h}\right),\left(\mathrm{P}_{f+h}\right)$ respectively, we have $\{0\}$.

CoRoLLARY 2.10. Under assumption $(A 1), \mathcal{S}_{f+h} \subset \mathcal{S}_{f h}$, and $\mathcal{S}_{f h}=\operatorname{cone}\left(\mathcal{S}_{f+h}\right) \backslash$

So far we know that under (A1) $\left(\mathrm{P}_{f+h}\right)$ has an optimal solution. The following proposition tells us that the point $x_{c}\left(d^{*}\right)$, where $d^{*}$ is an optimal solution to $\left(\mathrm{P}_{f+h}\right)$, is an interior point of $P$.

Proposition 2.11. If (A1) holds and $d^{*}$ is an optimal solution to $\left(\mathrm{P}_{f+h}\right)$, then the corresponding center $x_{c}\left(d^{*}\right)$ is an interior point of $P$.

Proof. By the KKT conditions, $d^{*}$ is optimal if and only if there are $\lambda^{T}=$ $\left(\lambda_{1}, \ldots, \lambda_{m}\right) \geq 0$ such that $\nabla F\left(d^{*}\right)=\lambda$, i.e.,

(12) $-\left(a_{i}^{T} x_{c}\left(d^{*}\right)-l_{i}\right)\left(a_{i}^{T} x_{c}\left(d^{*}\right)-u_{i}\right)=\frac{1}{n} \operatorname{det}\left(A D A^{T}\right)^{-\frac{1}{n}} a_{i}^{T}\left(A D A^{T}\right)^{-1} a_{i}+\lambda_{i}$

for all $i=1, \ldots, m$.

The right hand side of (12) is positive, hence $x_{c}\left(d^{*}\right)$ is an interior point.

The following example shows that the Hessian of $F$ might be only positive semidefinite, and the optimal solution set $\mathcal{S}_{f+h}$ can be a segment. 
Example: Let

$$
A=\left(\begin{array}{rrrr}
1 & 0 & 1 & -2 \\
0 & 1 & 1 & 1
\end{array}\right)
$$

and

$$
\begin{aligned}
& l=\left(-\sqrt{\frac{1}{2}},-\sqrt{\frac{1}{2}},-1,-\sqrt{\frac{5}{2}}\right)^{T}, \\
& u=\left(\sqrt{\frac{1}{2}}, \sqrt{\frac{1}{2}}, 1, \sqrt{\frac{5}{2}}\right)^{T} .
\end{aligned}
$$

Then, if we take $d^{0}=\left(\frac{1}{4}, \frac{5}{8}, \frac{1}{4}, \frac{1}{8}\right)^{T}, \nabla^{2} F\left(d^{0}\right)$ is singular. Indeed, $r=0$ so $x_{c}(d)=0$ for all $d$, whence $H_{f}(d)=0$. Also, it is easy to see that $(6,3,-2,-1)^{T}$ lies in the null space of $H_{h}$ (see (8)). Moreover, let

$$
\begin{aligned}
d & =\left(0, \frac{1}{2}, \frac{1}{3}, \frac{1}{6}\right)^{T}, \\
\bar{d} & =(1,1,0,0)^{T}, \quad \text { and } \\
d_{\lambda} & =\lambda d+(1-\lambda) \bar{d}, \quad \lambda \in[0,1] .
\end{aligned}
$$

Then,

$$
\begin{aligned}
F\left(d_{\lambda}\right) & =f\left(d_{\lambda}\right)+h\left(d_{\lambda}\right) \\
& =r^{T} D_{\lambda} A^{T}\left(A D_{\lambda} A^{T}\right)^{-1} A D_{\lambda} r-l^{T} D_{\lambda} u+\operatorname{det}\left(A D_{\lambda} A^{T}\right)^{-\frac{1}{n}} \\
& \equiv 2\left(\text { note that } r=0,-l^{T} D_{\lambda} u=1, A D_{\lambda} A^{T}=I\right),
\end{aligned}
$$

and $\nabla F\left(d_{\lambda}\right)=0$ (see Lemma 2.3). Therefore, the solution set of $\left(\mathrm{P}_{f+h}\right)$ is (at least) $\left\{d_{\lambda}=\lambda d+(1-\lambda) \bar{d} \mid \lambda \in[0,1]\right\}$.

We note that any point used as a "center" must be uniquely defined. Although the optimal solution set $\mathcal{S}_{f+h}$ may not be a singleton, it turns out that $x_{c}\left(d^{*}\right)$ is unique, so it can be defined as a "center". We now prove the uniqueness. We require several lemmas.

Lemma 2.12. Let $\mathcal{S}_{f+h}$ be the solution set to $\left(\mathrm{P}_{f+h}\right)$. Then there is a constant $\kappa_{f+h}$ such that for any $d \in \mathcal{S}_{f+h}$

$$
\left(\frac{u+l}{2}\right)^{T} D A^{T}\left(A D A^{T}\right)^{-1} A D\left(\frac{u+l}{2}\right)-l^{T} D u=\left(\operatorname{det}\left(A D A^{T}\right)\right)^{-\frac{1}{n}}=\kappa_{f+h} .
$$

Proof. Since $\mathcal{S}_{f+h}$ is a convex set, $F(d)=F\left(d^{\prime}\right), \forall d, d^{\prime} \in \mathcal{S}_{f+h}$; on the other hand, because $f$ and $h$ are nonnegative functions which are homogeneous with degrees 1 and -1 respectively, thus for $d \in \mathcal{S}_{f+h}, f(d)=h(d)=\frac{1}{2} F(d)$. The lemma follows immediately.

Lemma 2.13. If $\operatorname{det}(I+\varepsilon \Lambda) \equiv 1$, for all $\varepsilon \in(0, \eta)$, where $\eta$ is some positive number, and

$$
\Lambda=\operatorname{diag}\left(\lambda_{1}, \ldots, \lambda_{n}\right)
$$

then $\Lambda=0$. 
Proof. We have

$$
\operatorname{det}(I+\varepsilon \Lambda)=\prod_{i=1}^{n}\left(1+\varepsilon \lambda_{i}\right) \equiv 1, \quad \forall \varepsilon \in(0, \eta)
$$

Thus the coefficient of $\varepsilon^{n}$ should be zero, i.e., $\prod_{i=1}^{n} \lambda_{i}=0$, so, at least one of $\lambda_{i}$ 's is zero, say $\lambda_{n}=0$. Thus we have

$$
\operatorname{det}(I+\varepsilon \Lambda)=\prod_{i=1}^{n-1}\left(1+\varepsilon \lambda_{i}\right) .
$$

The same argument implies that at least one of $\left\{\lambda_{1}, \ldots, \lambda_{n-1}\right\}$ is zero; keep using this argument until eventually we have $\Lambda=0$. $\mathrm{B}$

Proposition 2.14. There is a constant matrix $M\left(\mathcal{S}_{f+h}\right)$ such that

$$
A D A^{T}=M\left(\mathcal{S}_{f+h}\right), \quad \forall d \in \mathcal{S}_{f+h} .
$$

Proof. We need only to show that for any $d, \bar{d} \in \mathcal{S}_{f+h}$, we have

$$
A D A^{T}=A \bar{D} A^{T} \text {. }
$$

By Lemma 2.12,

$$
\operatorname{det}\left(A(D+\varepsilon(\bar{D}-D)) A^{T}\right)=\operatorname{det}\left(A D A^{T}\right), \forall \varepsilon \in[0,1]
$$

On the other hand,

$$
\begin{gathered}
\operatorname{det}\left(A(D+\varepsilon(\bar{D}-D)) A^{T}\right)=\operatorname{det}\left(A D A^{T}+\varepsilon A(\bar{D}-D) A^{T}\right) \\
=\operatorname{det}\left(A D A^{T}\right)^{\frac{1}{2}} \operatorname{det}\left(I+\varepsilon\left(A D A^{T}\right)^{-\frac{1}{2}} A(\bar{D}-D) A^{T}\left(A D A^{T}\right)^{-\frac{1}{2}}\right) \operatorname{det}\left(A D A^{T}\right)^{\frac{1}{2}} .
\end{gathered}
$$

Thus, (14) implies

$$
\operatorname{det}\left(I+\varepsilon\left(A D A^{T}\right)^{-\frac{1}{2}} A(\bar{D}-D) A^{T}\left(A D A^{T}\right)^{-\frac{1}{2}}\right)=1 .
$$

By Lemma 2.13 , the eigenvalues of $\left(A D A^{T}\right)^{-\frac{1}{2}} A(\bar{D}-D) A^{T}\left(A D A^{T}\right)^{-\frac{1}{2}}$ are all zeros, so

$$
\left(A D A^{T}\right)^{-\frac{1}{2}} A(\bar{D}-D) A^{T}\left(A D A^{T}\right)^{-\frac{1}{2}}=0,
$$

i.e. $A(\bar{D}-D) A^{T}=0$. Hence $A \bar{D} A^{T}=A D A^{T}$. $\square$

Thus the minimum volume ellipsoids containing $P$ all have the same shape but possibly different locations. We then show, in the following, that the location is also unique.

Proposition 2.15. $A D r=A \bar{D} r, \quad \forall d, \bar{d} \in \mathcal{S}_{f+h}$.

Proof. From Lemma 2.12 and Proposition 2.14

$$
\begin{gathered}
r^{T}(D+\varepsilon(\bar{D}-D)) A^{T}\left(A D A^{T}\right)^{-1} A(D+\varepsilon(\bar{D}-D)) r-l^{T}(D+\varepsilon(\bar{D}-D)) u= \\
=r^{T} D A^{T}\left(A D A^{T}\right)^{-1} A D r-l^{T} D U, \quad \forall \varepsilon \in[0,1] .
\end{gathered}
$$

Thus by letting the coefficient of $\varepsilon^{2}$ be zero,

$$
r^{T}(\bar{D}-D) A^{T}\left(A D A^{T}\right)^{-1} A(\bar{D}-D) r=0 .
$$


Since $\left(A D A^{T}\right)^{-1}$ is positive definite,

$$
A(\bar{D}-D) r=0 \text {, i.e., } A \bar{D} r=A D r .
$$

Theorem 2.16 (Uniqueness). If $d^{*}$ solves $\left(\mathrm{P}_{f+h}\right), x_{c}\left(d^{*}\right)$ is unique.

Proof. By Propositions 2.14 and 2.15, for any $d \in \mathcal{S}_{f+h}$,

$$
x_{c}^{*}=\left(A D A^{T}\right)^{-1} \cdot A D r \text { is a constant vector } .
$$

Note: from Propositions 2.14 and 2.15 , if we find one optimal solution, say $d^{*}$, then the whole solution set can be expressed as

$\mathcal{S}_{f+h}=\left\{d \in \mathcal{D}: A D A^{T}=A D^{*} A^{T}, A D r=A D^{*} r, l^{T} D u=l^{T} D^{*} u\right\} \subset d^{*}+\left\{d: A D A^{T}=0\right\}$

2.2. Algorithms for $\left(\mathrm{P}_{f+h}\right)$. We now consider how to use model $\left(\mathrm{P}_{f+h}\right)$ to solve the original feasibility problem, and how to obtain the center $x_{c}^{*}:=x_{c}\left(d^{*}\right)$ (by the uniqueness theorem, this definition is well-defined).

The first method is a coordinate descent algorithm.

\section{Algorithm 2.1}

- Initialization. Choose $d^{0}>0$, and scale it so that $f\left(d^{0}\right)=h\left(d^{0}\right)$; set $k=0$.

- For $k=0,1, \ldots$, do If $x_{c}^{k}:=x_{c}\left(d^{k}\right) \in P$, stop with the feasible solution $x_{c}^{k}$; otherwise, choose $j$ with the $j$-th constraint violated by $x_{c}^{k}$. Let

$$
\beta:=\frac{s_{j}^{2}}{f\left(d^{k}\right) a_{j}^{T}\left(A D A^{T}\right)^{-1} a_{j}}, \quad \gamma:=a_{j}^{T}\left(A D A^{T}\right)^{-1} a_{j}
$$

and

$$
\left(l_{0}, u_{0}\right)= \begin{cases}\left(l_{j}, l_{j}+\left(f\left(d^{k}\right) \gamma\right)^{\frac{1}{2}}\right) & \text { if } a_{j}^{T} x_{c}<l_{j} \\ \left(u_{j}-\left(f\left(d^{k}\right) \gamma\right)^{\frac{1}{2}}, u_{j}\right) & \text { if } a_{j}^{T} x_{c}>u_{j}\end{cases}
$$

If $l_{0}>u_{j}$ or $u_{0}<l_{j}$, stop with the conclusion that the original system is not consistent.

(i) (canonical case) If $\beta \leq \frac{1}{4}$, we take $\lambda^{k}=\frac{1}{n \gamma}$, and $d^{k+1}=d^{k}+\lambda^{k} e_{j}$.

(ii) If $\beta>\frac{1}{4}$, we take $\lambda^{k}=\frac{1}{n \gamma}$, and $d^{k+1}=d^{k}+\lambda^{k} e_{j}$, AND update the bounds as follows:

$$
\begin{gathered}
l_{j} \leftarrow \frac{d_{j}^{k} l_{j}+\tilde{d}_{0}^{k+1} l_{0}}{d_{j}^{k}+\tilde{d}_{0}^{k+1}} \text { if } a_{j}^{T} x_{c}>u_{j}, \text { or } \\
u_{j} \leftarrow \frac{d_{j}^{k} u_{j}+\tilde{d}_{0}^{k+1} u_{0}}{d_{j}^{k}+\tilde{d}_{0}^{k+1}} \text { if } a_{j}^{T} x_{c}<l_{j}
\end{gathered}
$$

where $\tilde{d}_{0}^{k+1}=\frac{1}{n \gamma}$.

Then, scale $d^{k+1}$ so that $f\left(d^{k+1}\right)=h\left(d^{k+1}\right)$, where $f$ is computed with the updated $j$-th bounds; set $k \leftarrow k+1$, and repeat. 
The difference from the Liao and Todd algorithm [18] is that here the objective function is $F(d):=f(d)+h(d)$ instead of $v(d):=f(d) \cdot h(d)$ in the Liao and Todd algorithm.

THEOREM 2.17. Suppose that $\left\{d^{k}\right\}$ is the sequence generated by the algorithm. Then there is $\delta, 0<\delta<1$, depending only on $n$, such that

$$
\frac{F\left(d^{k+1}\right)}{F\left(d^{k}\right)} \leq \delta \text {, as long as } x_{c}\left(d^{k}\right) \notin P \text {. }
$$

Proof. Let $\tilde{d}^{k+1}$ be $d^{k}+\lambda^{k} e_{j}$ (the next iterate before scaling). Also let $\theta=\lambda \gamma$ and $\theta_{0}:=\frac{\left(a_{j}^{T} x_{c}-l_{j}\right)\left(a_{j}^{T} x_{c}-u_{j}\right)}{s_{j}^{2}} \geq 0$. Then the same analysis as in Liao and Todd [18] leads to

$$
\begin{aligned}
\frac{F\left(\tilde{d}^{k+1}\right)}{F\left(d^{k}\right)} & =1+\frac{1}{F\left(d^{k}\right)}\left[f\left(d^{k}\right) \beta \frac{\theta}{1+\theta}\left(\theta-\theta_{0}\right)-\left(1-(1+\theta)^{-\frac{1}{n}}\right) h\left(d^{k}\right)\right] \\
& =1-\frac{f\left(d^{k}\right)}{f\left(d^{k}\right)+h\left(d^{k}\right)}\left[1-(1+\theta)^{-\frac{1}{n}}-\beta \frac{\theta}{1+\theta}\left(\theta-\theta_{0}\right)\right] \\
& =1-\frac{1}{2}\left[1-(1+\theta)^{-\frac{1}{n}}-\beta \frac{\theta}{1+\theta}\left(\theta-\theta_{0}\right)\right] .
\end{aligned}
$$

Let

$$
R(\theta):=1-(1+\theta)^{-\frac{1}{n}}-\beta \frac{\theta}{1+\theta}\left(\theta-\theta_{0}\right)
$$

As in [18], it is enough to deal with the canonical case, i.e., $\beta \leq \frac{1}{4}$. Since $\theta_{0}>0$, so

$$
\begin{aligned}
R(\theta) & \geq 1-(1+\theta)^{-\frac{1}{n}}-\beta \frac{\theta^{2}}{1+\theta} \\
& \geq 1-(1+\theta)^{-\frac{1}{n}}-\frac{1}{4} \cdot \frac{\theta^{2}}{1+\theta}=: r(\theta) .
\end{aligned}
$$

The following inequality can be obtained from Taylor's Theorem:

$$
\left|(1+\theta)^{\frac{1}{n}}-\left(1+\frac{\theta}{n}\right)\right| \leq \frac{n-1}{2 n^{2}} \theta^{2}, \text { for } 0<\theta \leq 1 .
$$

Hence, noting that $\theta>0$,

$$
\left|(1+\theta)^{-\frac{1}{n}}-\left(1+\frac{\theta}{n}\right)^{-1}\right| \leq \frac{n-1}{2 n^{2}} \theta^{2} .
$$

Therefore, for $0<\theta \leq 1$,

$$
\begin{aligned}
r(\theta) & \geq 1-\left(1+\frac{\theta}{n}\right)^{-1}-\frac{1}{4} \cdot \frac{\theta^{2}}{1+\theta}-\frac{n-1}{2 n^{2}} \theta^{2} \\
& =\frac{\theta}{n+\theta}-\frac{1}{4} \cdot \frac{\theta^{2}}{1+\theta}-\frac{n-1}{2 n^{2}} \theta^{2} \\
& =\theta\left[\frac{4-n \theta+4 \theta-\theta^{2}}{4(n+\theta)(1+\theta)}-\frac{n-1}{2 n^{2}} \theta\right] .
\end{aligned}
$$


If we take $\theta=\frac{1}{n}$, we have

$$
\begin{aligned}
r(\theta) & \geq \frac{1}{n}\left[\frac{3}{4(1+\theta)(n+1)}-\frac{n-1}{2 n^{3}}\right] \\
& \geq \frac{1}{n}\left[\frac{3}{8(n+1)}-\frac{n-1}{2 n^{3}}\right] \\
& \geq \frac{1}{n}\left[\frac{6 n^{3}-8 n^{2}+8}{16 n^{3}(n+1)}\right] \\
& \geq \frac{1}{n} \cdot \frac{1}{8 n}=\frac{1}{8 n^{2}} .
\end{aligned}
$$

So

$$
\frac{F\left(\tilde{d}^{k+1}\right)}{F\left(d^{k}\right)}=1-\frac{1}{2} R(\theta) \leq 1-\frac{1}{2} r(\theta) \leq 1-\frac{1}{16 n^{2}} .
$$

Since $F\left(\tilde{d}^{k+1}\right) \leq F\left(d^{k}\right)$, the theorem follows by letting $\delta=1-\frac{1}{16 n^{2}}$. $\square$

In term of the shrinkage of the volumes of the corresponding ellipsoids, we have

$$
\frac{\operatorname{volume}\left(E^{+}\right)}{\operatorname{volume}(E)} \leq \delta^{n}=\left(1-\frac{1}{16 n^{2}}\right)^{n} \leq \exp \left(-\frac{1}{16 n}\right) .
$$

Although, from the above results, coordinate descent algorithm is polynomial, it converges slowly. Liao [17] shows that, although $F$ may not be strictly convex over $\mathcal{D}$ as shown earlier in an example, $F$ is strictly convex on $\Theta$ :

$$
\Theta:=R_{+}^{m} \cap<0>^{\perp}
$$

where $\langle 0\rangle^{\perp}$ is the orthogonal complement of $\left.<0\right\rangle$ and

$$
<0>=\left\{d: A D A^{T}=0\right\}=\{d: C d=0\}
$$

with $C=B \circ B$ where $B=A^{T} A$. Based on these observations Liao [17] proposes a partial Newton-step algorithm which has a stronger convergence property: under certain conditions, certain components of the iterates produced by this algorithm converge quadratically. However, the partial Newton-step algorithm is still not an efficient model because Newton's method cannot be used without restriction. In the next section we will propose another model where Newton's method can be employed completely.

When we use model I to solve the feasibility problem (1), there are three possible outcomes according to the values of $F_{\text {inf }}:=\inf \{F(d): d \in \mathcal{D}\}$.

THEOREM 2.18 .

(i) $F_{\text {inf }}>0$ : then there is $d^{*} \in \mathcal{D}$ such that $F\left(d^{*}\right)=F_{\text {inf }}$ and $x_{c}\left(d^{*}\right)$ is feasible;

(ii) $F_{\text {inf }}<0$ : then $P=\emptyset$;

(iii) $F_{\text {inf }}=0$ : then $\operatorname{int}(P)=\emptyset$ but $P \neq \emptyset$.

Proof. It is easy to see that if we replace (A1) by $F_{\text {inf }}>0$ then $f$ has no direction of recession and so the conclusions of Theorem 2.8 still hold. (i) thus follows. If $F_{\text {inf }}<0$, then there is $d \in \mathcal{D}$ such that $F(d)<0$ and therefore $f(d)<0$. Hence, by Theorem 2.7, $P=\emptyset$, which shows (ii). (iii) follows from Theorem 2.8 and Theorem 2.7.

In case (iii), if there is $d^{*} \in \mathcal{D}$ at which $f\left(d^{*}\right)=0$, then $x_{c}\left(d^{*}\right)$ is feasible; however, in general, $x_{c}\left(d^{k}\right)$ does not approach the feasible set even if $d^{k}$ is such that $F\left(d^{k}\right) \longrightarrow 0$ as shown in the following example: 
Example: Let

$$
\left(\begin{array}{l}
0 \\
0 \\
2 \\
0
\end{array}\right) \leq\left(\begin{array}{ll}
1 & 0 \\
0 & 1 \\
1 & 0 \\
0 & 1
\end{array}\right) x \leq\left(\begin{array}{c}
2 \\
2 \\
4 \\
30
\end{array}\right) .
$$

By calculation, we have

$$
\begin{aligned}
x_{c} & =\left(\frac{d_{1}+3 d_{3}}{d_{1}+d_{3}}, \frac{d_{2}+15 d_{4}}{d_{2}+d_{4}}\right), \\
f(d) & =\frac{\left(d_{1}+3 d_{3}\right)^{2}}{d_{1}+d_{3}}+\frac{\left(d_{2}+15 d_{4}\right)^{2}}{d_{2}+d_{4}}-8 d_{3}, \\
h(d) & =\frac{1}{\sqrt{\left(d_{1}+d_{3}\right)\left(d_{2}+d_{4}\right)}} .
\end{aligned}
$$

If we take $d_{1}^{k}=d_{3}^{k}=\frac{1}{2}$ and $d_{2}^{k}=d_{4}^{k}=\frac{1}{k}$, then $v\left(d^{k}\right) \longrightarrow 0$ as $k \longrightarrow \infty$, but $x_{c}\left(d^{k}\right) \longrightarrow(2,8)^{T}$ which is not feasible. By scaling, this sequence of $d^{k}$ can be converted to $\bar{d}^{k}$ such that $F\left(\bar{d}^{k}\right) \longrightarrow 0$ as $k \longrightarrow \infty$ and $x_{c}\left(\bar{d}^{k}\right)=x_{c}\left(d^{k}\right) \longrightarrow(2,8)^{T}$.

口

3. Model II and the associated center. Model I provides us with a kind of center of $P$, but this center is not so easy to obtain practically. In this section we propose a simpler model whose associated center can be obtained more efficiently.

By examining the previous model, we find that it is based on the homogeneous properties of $f$ and $h$. Since $f$ provides a tool for determining the feasibility of our problem we want to keep it, but the role of function $h$ is really like a barrier: it balances the weights $d_{i}, i=1, \ldots, m$, and forces the corresponding $x(d)$ to approach the center $x_{c}^{*}$. We will use a much simpler function to replace $h$. The new model is more efficient in practice.

As a matter of fact, any convex homogeneous function $B$ of degree -1 defined on $\mathcal{D}$ such that

(i) $f+B$ has an optimal solution in $\mathcal{D}$, and

(ii) $\nabla B(d) \leq 0$ for any $d \in \mathcal{D}$,

can play the same role as $h$ does.

One such function, which we choose for our new model, is as follows:

$$
B(d):=e^{T} d^{-1}:=\sum_{i=1}^{m} \frac{1}{d_{i}} .
$$

We let $B(d)=e^{T} d^{-1}=+\infty$ if any $d_{i}=0$.

The corresponding model, defined as Model II, is

$$
\begin{array}{ll}
\min & F^{I I}(d):=f(d)+B(d) \quad\left(\mathrm{P}_{f+B}\right) \\
\text { s.t. } d \in \mathcal{D}
\end{array}
$$

where

$$
\begin{aligned}
f(d) & :=x_{c}^{T} A D A^{T} x_{c}-l^{T} D u, \\
x_{c}(d) & :=\left(A D A^{T}\right)^{-1} A D r \\
B(d) & :=e^{T} d^{-1} \\
\mathcal{D} & :=\left\{d: d \geq 0,\left(A D A^{T}\right) \text { is nonsingular }\right\} .
\end{aligned}
$$

We note that $\left(\mathrm{P}_{f+B}\right)$ is equivalent to minimizing $F^{I I}(d)$ over $\mathcal{D}_{+}:=\{d>0: d \in \mathcal{D}\}$. 
3.1. Properties of $\left(\mathrm{P}_{f+B}\right)$. Obviously, $B(d)$ is a strictly convex function, so $F^{I I}(d)$ is strictly convex too. Accordingly, we have

THEOREM 3.1. If (A1) holds, then $F^{I I}(d)$ is a closed proper strictly convex function, and $F^{I I}(d)$ has no direction of recession. Thus there is an unique solution to $\left(\mathrm{P}_{f+B}\right)$; moreover, the level set

$$
\mathcal{D}_{e}^{I I}:=\left\{d \in \mathcal{D}: F^{I I}(d) \leq F^{I I}(e)\right\}
$$

is a closed bounded convex set.

Proof. Similar to that in the previous section. We just note that, under assumption (A1), $f(d)$ has no recession direction in $R_{+}^{m}$, hence $F^{I I}(d)$ has no recession direction either. $\square$

It is easy to prove that if $d^{*}$ is the unique solution to $\left(\mathrm{P}_{f+B}\right)$, the associated $x_{c}\left(d^{*}\right)$ is an interior point of $P$. We thus define $x_{c}\left(d^{*}\right)$ as the "center" associated with model II. In the following we show that $\left(\mathrm{P}_{f+B}\right)$ is equivalent to finding a minimum volume ellipsoid among those with a particular shape, and $x_{c}\left(d^{*}\right)$ is the center of this kind of ellipsoid.

First we note that $\left(\mathrm{P}_{f+B}\right)$ is equivalent to

$$
\begin{aligned}
& \min v^{I I}(d):=f(d) \cdot B(d) \\
& \text { s.t. } d \in \mathcal{D}
\end{aligned}
$$

in the following sense: if $d^{*}$ is the solution to $\left(\mathrm{P}_{f+B}\right)$, it is also a solution to $\left(\mathrm{P}_{f B}\right)$; and if $d^{*}$ is a solution to $\left(\mathrm{P}_{f B}\right)$, then $\sqrt{\frac{h\left(d^{*}\right)}{f\left(d^{*}\right)}} d^{*}$ is the unique solution to $\left(\mathrm{P}_{f+B}\right)$.

Lemma 3.2. Suppose that $d_{i} \geq 0, i=1, \ldots, m$. Then

$$
\sum_{i=1}^{m} d_{i} w_{i}^{2} \leq 1
$$

implies

$$
\sum_{i=1}^{m} w_{i}^{2} \leq e^{T} d^{-1}
$$

Proof. If there is $i_{0}$ such that $d_{i_{0}}=0$, then (17) is always true, since we have set $e^{T} d^{-1}$ to $\infty$ in this case; otherwise we suppose that $d_{i_{0}}=\min \left\{d_{i}: i=1, \ldots, m\right\}$. Then

$$
d_{i_{0}} \sum_{i=1}^{m} w_{i}^{2} \leq \sum_{i=1}^{m} d_{i} w_{i}^{2} \leq 1
$$

which leads to

$$
\sum_{i=1}^{m} w_{i}^{2} \leq \frac{1}{d_{i_{0}}} \leq e^{T} d^{-1} .
$$

We define

$$
E^{I I}(d):=\left\{x \in R^{n}: \frac{1}{e^{T} d^{-1}}\left(x-x_{c}\right)^{T} A A^{T}\left(x-x_{c}\right) \leq f(d)\right\}
$$


where

$$
\begin{gathered}
x_{c}=x_{c}(d)=\left(A D A^{T}\right)^{-1} A D r, \\
f(d)=x_{c}^{T} A D A^{T} x_{c}-l^{T} D u .
\end{gathered}
$$

Then $\left(\mathrm{P}_{f B}\right)$ is equivalent to minimizing the volume of the ellipsoid $E^{I I}(d)$.

The relationship between this ellipsoid and the one defined by (4) is described in the following proposition.

Proposition 3.3. If $E(d)$ is the ellipsoid defined by (4), then $E(d) \subset E^{I I}(d)$.

Proof.

$$
\begin{aligned}
E(d) & =\left\{x \in R^{n}:\left(x-x_{c}\right)^{T} A D A^{T}\left(x-x_{c}\right) \leq f(d)\right\} \\
& =\left\{x \in R^{n}:\left(A^{T}\left(x-x_{c}\right)\right)^{T} D\left(A^{T}\left(x-x_{c}\right)\right) \leq f(d)\right\} .
\end{aligned}
$$

Let $w:=A^{T}\left(x-x_{c}\right)$. Then by Lemma (3.2)

$$
\begin{aligned}
E(d) & =\left\{x \in R^{n}: w^{T} D w \leq f(d)\right\}=\left\{x \in R^{n}: \sum_{i=1}^{m} d_{i} w_{i}^{2} \leq f(d)\right\} \\
& \subseteq\left\{x \in R^{n}: \sum_{i=1}^{m} w_{i}^{2} \leq f(d) e^{T} d^{-1}\right\} \\
& =\left\{x \in R^{n}: \frac{1}{e^{T} d^{-1}}\left(x-x_{c}\right)^{T} A A^{T}\left(x-x_{c}\right) \leq f(d)\right\} \\
& =E^{I I}(d) .
\end{aligned}
$$

Since $P \subset E(d)$, the above result also shows that $P \subset E^{I I}(d)$.

We note that since $A D A^{T}$ can be nonsingular if one component of $d$ is zero, thus the volume of $E(d)$ is finite, but for this $d$ the volume of $E^{I I}(d)$ is infinite which shows that $E^{I I}$ and $E$ are not equivalent, i. e. there is no constant $\lambda>0$ such that

$$
E(d) \subset E^{I I}(d) \subset \lambda E(d) \text {, for all } d \in \mathcal{D} .
$$

3.2. Algorithms for $\left(\mathrm{P}_{f+B}\right)$. We describe first a coordinate descent algorithm to solve the feasibility problem $(P)$ by using model II.

\section{Algorithm 3.1}

- Initialization. Normalize the constraints so that $\left\|a_{i}\right\|=1, \forall i=1, \ldots, m$. Choose $d^{0}>0$ and scale it so that $f\left(d^{0}\right)=B\left(d^{0}\right)$; set $k=0$.

- For $k=0,1, \ldots$, do

If $x_{c}^{k}:=x_{c}\left(d^{k}\right) \in P$, stop with the feasible solution $x_{c}^{k}$; otherwise, choose $j$ with the $j$-th constraint violated by $x_{c}^{k}$. Then take $\lambda^{k}=\arg \min \left\{F^{I I}\left(d^{k}+\right.\right.$ $\left.\left.\lambda e_{j}\right): \lambda \geq 0\right\}$, and $d^{k+1}=d^{k}+\lambda^{k} e_{j}$; scale $d^{k+1}$ so that $f\left(d^{k+1}\right)=B\left(d^{k+1}\right)$; set $k \leftarrow k+1$, and repeat.

To analyze this algorithm we first need the following result.

Lemma 3.4. Suppose that the feasible region $P$ of (1) is "fat", so that there is a ball, say $\mathcal{B}$, of radius $\delta_{0}$ contained in $P$, i.e., there some $\hat{x}$ such that $\mathcal{B}=\mathcal{B}\left(\hat{x} ; \delta_{0}\right) \subset P$, 
and $\left\|a_{i}\right\|=1, \forall i=1, \ldots, m$. Then, for all $i=1, \ldots, m$ and $d \in \mathcal{D}_{+}$with $f(d)=B(d)$ and $F^{I I}(d) \leq F^{I I}(e)$

$$
\begin{aligned}
& \kappa_{i}(d):=\frac{f(d)}{a_{i}^{T}\left(A D A^{T}\right)^{-1} a_{i}} \leq \frac{\left(F_{e}^{I I}\right)^{2}}{4 \delta_{0}^{2}} \\
& \beta_{i}(d):=\frac{s_{i}^{2}}{f(d) a_{i}^{T}\left(A D A^{T}\right)^{-1} a_{i}} \leq \frac{s_{\max }^{2}}{\delta_{0}^{2}}
\end{aligned}
$$

where

$$
\begin{aligned}
F_{e}^{I I} & :=F^{I I}(e)=\left(\frac{u+l}{2}\right)^{T} A^{T}\left(A A^{T}\right)^{-1} A\left(\frac{u+l}{2}\right)-l^{T} u+m, \\
s_{\max } & :=\max \left\{s_{i}: i=1, \ldots, m\right\} .
\end{aligned}
$$

Proof. Since $2 \sqrt{f(d) a_{i}^{T}\left(A D A^{T}\right)^{-1} a_{i}}$ is the width of the ellipsoid $E(d)$ in the direction $a_{i}$, so

$$
\sqrt{f(d) a_{i}^{T}\left(A D A^{T}\right)^{-1} a_{i}} \geq \delta_{0}
$$

Thus

$$
\begin{aligned}
\kappa_{i}(d) & =\frac{f(d)}{a_{i}^{T}\left(A D A^{T}\right)^{-1} a_{i}}=\frac{f(d)^{2}}{f(d) a_{i}^{T}\left(A D A^{T}\right)^{-1} a_{i}} \\
& =\frac{F^{I I}(d)^{2}}{4 f(d) a_{i}^{T}\left(A D A^{T}\right)^{-1} a_{i}} \leq \frac{\left(F^{I I}(e)\right)^{2}}{4 f(d) a_{i}^{T}\left(A D A^{T}\right)^{-1} a_{i}} \leq \frac{\left(F_{e}^{I I}\right)^{2}}{4 \delta_{0}^{2}} .
\end{aligned}
$$

On the other hand,

$$
\beta_{i}(d)=\frac{s_{i}^{2}}{f(d) a_{i}^{T}\left(A D A^{T}\right)^{-1} a_{i}} \leq \frac{s_{\max }^{2}}{\delta_{0}^{2}}
$$

THEOREM 3.5. There is $\delta=\delta(n, \omega)$ where $\omega:=\max \left\{\frac{\left(F_{e}^{I I}\right)^{2}}{4 \delta_{0}^{2}}, \frac{s_{\text {max }}^{2}}{\delta_{0}^{2}}, 1\right\}, 0<\delta<1$, such that

$$
\frac{F^{I I}\left(d^{k+1}\right)}{F^{I I}\left(d^{k}\right)} \leq \delta \text {, as long as } x_{c}\left(d^{k}\right) \notin P .
$$

Proof. For simplicity we write $d$ for $d^{k}, \lambda$ for $\lambda^{k}$ and $d^{+}=d+\lambda^{k} e_{j}$. Then

$$
B\left(d^{+}\right)=B(d)-\frac{\lambda}{d_{j}\left(d_{j}+\lambda\right)}
$$

and from [18]

$$
f\left(d^{+}\right)=f(d)+f(d) \frac{\beta_{j} \theta}{1+\theta}\left(\theta-\theta_{0}\right) \leq f(d)\left(1+\frac{\beta_{j} \theta^{2}}{1+\theta}\right),
$$

where $\beta_{j}=\beta_{j}(d), \theta=\lambda \gamma_{j}, \gamma_{j}=a_{j}^{T}\left(A D A^{T}\right)^{-1} a_{j}$ and $\theta_{0}:=\frac{\left(a_{j}^{T} x_{c}-l_{j}\right)\left(a_{j}^{T} x_{c}-u_{j}\right)}{s_{j}^{2}} \geq 0$.

Also,

$$
\begin{aligned}
\sum d_{i} a_{i}^{T}\left(A D A^{T}\right)^{-1} a_{i}= & \operatorname{tr}\left(D A^{T}\left(A D A^{T}\right)^{-1} A\right) \\
= & \operatorname{tr}\left(A D A^{T}\left(A D A^{T}\right)^{-1}\right)=n \\
& 17
\end{aligned}
$$


so $d_{j} \gamma \leq n$. Thus, noting that $f(d)=B(d)$,

$$
\begin{aligned}
\frac{F^{I I}\left(d^{+}\right)}{F^{I I}(d)} & \leq 1-\frac{1}{2}\left[\frac{\lambda}{f(d) d_{j}\left(d_{j}+\lambda\right)}-\beta_{j} \frac{\theta^{2}}{1+\theta}\right] \\
& \leq 1-\frac{1}{2}\left[\frac{\theta}{n f(d)\left(d_{j}+\lambda\right)}-\beta_{j} \frac{\theta^{2}}{1+\theta}\right] \\
& \leq 1-\frac{1}{2}\left[\frac{\theta}{n \kappa_{j}(d)(n+\theta)}-\beta_{j} \frac{\theta^{2}}{1+\theta}\right] \\
& \leq 1-\frac{1}{2}\left[\frac{\theta}{n \omega(n+\theta)}-\omega \frac{\theta^{2}}{1+\theta}\right] .
\end{aligned}
$$

If we take $\theta=\frac{1}{\omega^{2} n^{2}}$, then

$$
\frac{F^{I I}\left(d^{+}\right)}{F^{I I}(d)} \leq 1-\frac{1}{16 n^{6} \omega^{5}}
$$

The theorem follows if we take $\delta=1-\frac{1}{16 n^{6} \omega^{5}}$. $\square$

In terms of the shrinkage of the volumes of the corresponding ellipsoids we have

$$
\frac{\operatorname{volume}\left(E^{I I}\left(d^{+}\right)\right)}{\operatorname{volume}\left(E^{I I}(d)\right)} \leq \exp \left(-\frac{1}{16 n^{5} \omega^{5}}\right)
$$

This shrinkage rate depends on $\omega$ and hence on the bound on $\kappa_{i}(d)$. We note that if the initial point is chosen as $d^{0}=\lambda e$ such that $F^{I I}\left(d^{0}\right)=2 f\left(d^{0}\right)=2 B\left(d^{0}\right)$, then we can obtain a simpler bound. We denote

$$
\operatorname{Lev}_{0}^{I I}:=\left\{d: F^{I I}(d) \leq F^{I I}\left(d^{0}\right)\right\} .
$$

Then, for $d \in \operatorname{Lev}_{0}^{I I}$ with $f(d)=B(d)$,

$$
\begin{aligned}
\kappa_{i}(d) & =\frac{f(d)^{2}}{f(d) a_{i}^{T}\left(A D A^{T}\right)^{-1} a_{i}}=\frac{\left(\frac{F^{I I}(d)}{2}\right)^{2}}{f(d) a_{i}^{T}\left(A D A^{T}\right)^{-1} a_{i}} \\
& \leq \frac{\left(\frac{F^{I I}\left(d^{0}\right)}{2}\right)^{2}}{f(d) a_{i}^{T}\left(A D A^{T}\right)^{-1} a_{i}}=\frac{f\left(d^{0}\right)^{2}}{f(d) a_{i}^{T}\left(A D A^{T}\right)^{-1} a_{i}} \\
& \leq \frac{f\left(d^{0}\right) B\left(d^{0}\right)}{\delta_{0}^{2}} \leq \zeta^{\frac{2}{n}} \cdot \operatorname{det}\left(A A^{T}\right)^{\frac{1}{n}}
\end{aligned}
$$

where $\zeta=\frac{\operatorname{volume}\left(E_{0}^{I I}\right)}{\operatorname{volume}(\mathcal{B})}$ which is the ratio of the volume of the initial ellipsoid over the volume of the ball contained in the feasible region $P$.

By scaling and changing variables we can assume, without loss of generality, $A A^{T}=I$. Thus Algorithm 3.1 is a kind of "ball method" [4] for which Todd [28] and Goffin [9] show that an exponential number of iterations may be required. But here, unlike the "ball method", we can use Newton's method to solve $\left(\mathrm{P}_{f+B}\right)$ thus getting a quadratic convergence rate; this follows from Theorem 3.1, since the Hessian of $F^{I I}$ is bounded in the compact level set.

We now state Newton's method for solving $\left(\mathrm{P}_{f+B}\right)$ in the following:

\section{Algorithm 3.2}

- Initialization. Take $d^{0}=e$ as the initial point; $k=0$ 
- For $k=0,1, \ldots$

If a convergence condition holds, stop. Otherwise, perform a line search along the Newton direction, that is: take

$$
d^{k+1}=d^{k}+\lambda^{k} d_{n t}
$$

where $d_{n t}:=-\nabla^{2} F^{I I}\left(d^{k}\right) \cdot \nabla F^{I I}\left(d^{k}\right)$, and

$$
\lambda^{k}=\arg \min \left\{F^{I I}\left(d^{k}+\lambda d_{n t}\right): \lambda \geq 0\right\} .
$$

Set $k=k+1$ and repeat.

If we use the above algorithm for solving the feasibility problem, we just set the condition of convergence as " $x_{c}\left(d^{k}\right) \in P$ ". Then, under (A1), it solves the feasibility problem (1) in finitely many iterations, since the Hessian of $F^{I I}\left(d^{k}\right)$ is bounded; indeed, as long as $x_{c}\left(d^{k}\right)$ is not feasible, we can get a constant decrease in $F^{I I}$ at each iteration, as in (19) below.

Note that $f(d)$ and $B(d)$ are homogeneous functions of degree 1 and -1 respectively. We can thus use a scaling technique to get a Newton-scaling algorithm:

\section{Algorithm 3.3}

- Initialization. Take $\tilde{d}^{0}=e$ and scale it to $d^{0}$, i.e., $d^{0}=\mu^{0} \tilde{d}^{0}$ with $\mu^{0}=$ $\sqrt{\frac{B\left(\tilde{d}^{0}\right)}{f\left(\tilde{d}^{0}\right)}} ;$ set $k=0$.

- For $k=0,1, \ldots$

If a convergence condition holds, stop. Otherwise, perform a line search along the Newton direction, that is: take

$$
\tilde{d}^{k+1}=d^{k}+\lambda^{k} d_{n t}
$$

where $d_{n t}:=-\nabla^{2} F^{I I}\left(d^{k}\right) \cdot \nabla F^{I I}\left(d^{k}\right)$, and

$$
\lambda^{k}=\arg \min \left\{F^{I I}\left(d^{k}+\lambda d_{n t}\right): \lambda \geq 0\right\} .
$$

Let

$$
d^{k+1}=\mu^{k+1} \tilde{d}^{k+1}
$$

with $\mu^{k+1}=\sqrt{\frac{B\left(\tilde{d}^{k+1}\right)}{f\left(\tilde{d}^{k+1}\right)}}$. Set $k=k+1$ and repeat.

As for the convergence property of this algorithm we have the following theorem.

THEOREM 3.6. Suppose (A1) holds, that is, $\operatorname{int}(P) \neq \emptyset$, and $\left\{d^{k}\right\}$ is the sequence of points generated by Algorithm 3.3. Then $d^{k}$ converges to $d^{*} q$-quadratically; what is more, Algorithm 3.3 solves the feasibility problem (1) in finitely many iterations.

Proof. Since Algorithm 3.3 is a descent method, by Theorem 3.1 we can restrict our attention to a compact set $\mathcal{D}_{e}^{I I}$. Let

$$
\begin{aligned}
\lambda_{u} & :=\max \left\{\text { largest eigenvalue of } H(d): d \in \mathcal{D}_{e}^{I I}\right\} \\
\lambda_{l} & :=\min \left\{\text { smallest eigenvalue of } H(d): d \in \mathcal{D}_{e}^{I I}\right\},
\end{aligned}
$$

where $H(d)$ is the Hessian of $F^{I I}$. Let $H$ denote $H\left(d^{k}\right)$ and $\nabla F$ denote $\nabla F^{I I}\left(d^{k}\right)$. Then for any $t>0$ there is $d(\xi):=d^{k}+\xi d_{n t}$ with $0<\xi<t$ such that

$$
F^{I I}\left(d^{k+1}\right) \leq F^{I I}\left(\tilde{d}^{k+1}\right) \leq F^{I I}\left(d^{k}+t d_{n t}\right)
$$




$$
\begin{aligned}
& =F^{I I}\left(d^{k}\right)+t \nabla F\left(d^{k}\right)^{T} d_{n t}+\frac{t^{2}}{2} d_{n t}^{T} H(d(\xi)) d_{n t} \\
& \leq F^{I I}\left(d^{k}\right)+t \nabla F\left(d^{k}\right)^{T} d_{n t}+\frac{t^{2}}{2} \lambda_{u}\left\|d_{n t}\right\|^{2} \\
& \leq F^{I I}\left(d^{k}\right)-t \nabla F\left(d^{k}\right)^{T} H^{-1} \nabla F\left(d^{k}\right)^{T}+\frac{t^{2}}{2} \lambda_{u}\left\|H^{-1} \nabla F\right\|^{2} \\
& \leq F^{I I}\left(d^{k}\right)-t \lambda_{u}^{-1}\|\nabla F\|^{2}+\frac{t^{2}}{2} \lambda_{u} \lambda_{l}^{-2}\|\nabla F\|^{2} \\
& =F^{I I}\left(d^{k}\right)-\sigma\|\nabla F\|^{2} \quad\left(\text { for } t=\lambda_{u}^{-2} \lambda_{l}^{2}\right)
\end{aligned}
$$

where $\sigma:=\frac{1}{2} \lambda_{u}^{-3} \lambda_{l}^{2}$. Thus $d^{k}$ must converge to $d^{*}$ for otherwise $F^{I I}\left(d^{k}\right) \longrightarrow-\infty$ contradicting (A1). On the other hand, from Theorem 3.1, there is $\bar{d}>0$ such that

$$
d_{i} \leq \bar{d}, \text { for all } d \in \mathcal{D}_{e}^{I I} \text { and all } i .
$$

If $\|\nabla F(d)\| \leq \bar{d}^{-2}$, then, for $i=1,2, \ldots, m$,

$$
-\left(a_{i}^{T} x_{c}(d)-l_{i}^{k}\right)\left(a_{i}^{T} x_{c}(d)-u_{i}^{k}\right)-\frac{1}{d_{i}^{2}} \geq-\frac{1}{\bar{d}^{2}}
$$

thus

$$
-\left(a_{i}^{T} x_{c}(d)-l_{i}^{k}\right)\left(a_{i}^{T} x_{c}(d)-u_{i}^{k}\right) \geq \frac{1}{d_{i}^{2}}-\frac{1}{d^{2}} \geq 0
$$

which in turn shows that

$$
l_{i}^{k} \leq a_{i}^{T} x_{c}(d) \leq u_{i}^{k}, \forall i=1,2, \ldots, m,
$$

and thus $x_{c}(d)$ is feasible for (FP). Therefore, by (19), $x_{c}\left(d^{k}\right)$ must be feasible to (FP) for $k \geq \frac{F\left(d^{0}\right) \bar{d}^{4}}{\sigma}-1$. Hence, the algorithm solves the feasibility problem (1) in finitely many iterations.

Since for any $k, \tilde{d}^{k}$ is the result of one step of Newton's method, it is easy to see that there is a constant, say $\gamma>0$, such that

$$
\left\|\tilde{d}^{k+1}-d^{*}\right\| \leq \gamma\left\|d^{k}-d^{*}\right\|^{2} .
$$

On the other hand, it can be shown by Taylor's theorem that, for all $d \in \mathcal{D}_{e}^{I I}$,

$$
\frac{1}{2} \lambda_{l}\left\|d-d^{*}\right\|^{2} \leq F^{I I}(d)-F^{I I}\left(d^{*}\right) \leq \frac{1}{2} \lambda_{u}\left\|d-d^{*}\right\|^{2} .
$$

Thus,

$$
\frac{\lambda_{l}}{2}\left\|d^{k+1}-d^{*}\right\|^{2} \leq F^{I I}\left(d^{k+1}\right)-F^{I I}\left(d^{*}\right) \leq F^{I I}\left(\tilde{d}^{k+1}\right)-F^{I I}\left(d^{*}\right) \leq \frac{\lambda_{u}}{2}\left\|\tilde{d}^{k+1}-d^{*}\right\|^{2}
$$

which implies

$$
\left\|d^{k+1}-d^{*}\right\|^{2} \leq \frac{\lambda_{u}}{\lambda_{l}}\left\|\tilde{d}^{k+1}-d^{*}\right\|^{2} .
$$

The theorem thus follows by (20).

As in model I, when model II is used for solving the feasibility problem (1), there are three possible outcomes according to the values of $F_{i n f}^{I I}:=\inf \left\{F^{I I}(d): d \in R_{++}^{m}\right\}$ where $R_{++}^{m}:=\left\{d \in R^{m}: d>0\right\}$. 
THEOREM 3.7.

(i) If $F_{\text {inf }}^{I I}>0$, then there is $d^{*} \in R_{++}^{m}$ such that $F^{I I}\left(d^{*}\right)=F_{\text {inf }}^{I I}$ and $x_{c}\left(d^{*}\right)$ is feasible;

(ii) If $F_{\text {inf }}^{I I}<0$, then $P=\emptyset$;

(iii) If $F_{\text {inf }}^{I I}=0$ then $\operatorname{int}(P)=\emptyset$ but $P \neq \emptyset$.

Proof. Similar to that of Theorem 2.18. $\square$

The example in the last part of the previous section can be adapted here to show that $x_{c}\left(d^{k}\right)$ may not approach the feasible region even if $d^{k}$ is such that $F^{I I}\left(d^{k}\right) \longrightarrow$ 0 . We should mention that this phenomenon did not occur in our computational experiments; in particular, our computational results always gave a feasible solution for this example.

We note that Liao [17] discusses some other possible models for solving the feasibility problem (1).

3.3. Computational results. In this section we discuss our computational experience with Algorithm 3.3. Obviously, the main computational effort is spent in calculating $\left(A D A^{T}\right)^{-1}$. If coordinate descent methods are used, it can be obtained by the rank-1 update formula, i.e. the Sherman-Morrison-Woodbury formula. For the general case, instead of calculating $\left(A D A^{T}\right)^{-1}$ directly, an equivalent linear system is solved. There are many methods for solving linear systems, see, for example, Golub and Van Loan [11]. Usually, some decomposition methods are used to reduce the linear system to a simpler system and the solution of the original system can be obtained from that of this simpler system. We would like to mention here three possible decomposition methods. The first one is the Cholesky Decomposition since $A D A^{T}$ is positive definite. A typical algorithm will give the Cholesky Decomposition with about $\frac{n^{3}}{6}$ flops (floating point operations: one multiply/divide and one add/subtract). The second possible choice is LU decomposition; a typical Gaussian elimination algorithm for computing the factorization $A D A^{T}=L U$ requires about $\frac{n^{3}}{3}$ flops. In either case, forming $A D A^{T}$ takes about $\frac{1}{2} m n^{2}$ flops. The third method is QR decomposition; using Householder orthogonalization, the QR decomposition of $D^{\frac{1}{2}} A^{T}$ needs $n^{2}\left(m-\frac{n}{3}\right)$ flops. Among these three possible choices the Cholesky Decomposition seems the best. Our computer code is written in MATLAB([19], [7]) and uses LU decomposition because MATLAB finds $x=A \backslash b$ by using the LU decomposition for $A$. This makes the code simpler yet will not affect the statistical analysis much, since we are mainly interested in the number of iterations and the time is a constant multiple of that using Cholesky factorization.

There is an example in Bland, Goldfarb and Todd [4], showing that convergence of the standard ellipsoid algorithm can be extremely slow. The example is $A^{T}=I, l=$ $u=0$; by using our models, as well as Burrell and Todd's algorithm [5], the solution can be obtained in just one iteration. This comparison might be not appropriate, but it does show the difference between our model and the ellipsoid method.

In the following we investigate the numerical behavior of model II by using the Newton-scaling algorithm, i.e., Algorithm 3.3. Specifically we will investigate the effects of $n, m, l$ and $u$ on the number of iterations and the time that is needed to get the first feasible solution. Here we have 4 factors, so a suitable model is the Graeco-Latin square design. The idea of this design can be found, for example, in Devore [8] and Bethea, Duran and Boullion [3]. Usually, the use of the Graeco-Latin square design can obtain a tremendous saving in time and money in that the total number of observations is greatly reduced. But this design requires no interactions 


$\begin{array}{lllllllll}\mathrm{A} \alpha & \mathrm{B} \gamma & \mathrm{C} \epsilon & \mathrm{D} \zeta & \mathrm{E} \theta & \mathrm{F} \beta & \mathrm{G} \delta & \mathrm{H} \varepsilon & \mathrm{I} \eta \\ \mathrm{B} \beta & \mathrm{C} \delta & \mathrm{D} \varepsilon & \mathrm{E} \eta & \mathrm{F} \alpha & \mathrm{G} \gamma & \mathrm{H} \epsilon & \mathrm{I} \zeta & \mathrm{A} \theta \\ \mathrm{C} \gamma & \mathrm{D} \epsilon & \mathrm{E} \zeta & \mathrm{F} \theta & \mathrm{G} \beta & \mathrm{H} \delta & \mathrm{I} \varepsilon & \mathrm{A} \eta & \mathrm{B} \alpha \\ \mathrm{D} \delta & \mathrm{E} \varepsilon & \mathrm{F} \eta & \mathrm{G} \alpha & \mathrm{H} \gamma & \mathrm{I} \epsilon & \mathrm{A} \zeta & \mathrm{B} \theta & \mathrm{C} \beta \\ \mathrm{E} \epsilon & \mathrm{F} \zeta & \mathrm{G} \theta & \mathrm{H} \beta & \mathrm{I} \delta & \mathrm{A} \varepsilon & \mathrm{B} \eta & \mathrm{C} \alpha & \mathrm{D} \gamma \\ \mathrm{F} \varepsilon & \mathrm{G} \eta & \mathrm{H} \alpha & \mathrm{I} \gamma & \mathrm{A} \epsilon & \mathrm{B} \zeta & \mathrm{C} \theta & \mathrm{D} \beta & \mathrm{E} \delta \\ \mathrm{G} \zeta & \mathrm{H} \theta & \mathrm{I} \beta & \mathrm{A} \delta & \mathrm{B} \varepsilon & \mathrm{C} \eta & \mathrm{D} \alpha & \mathrm{E} \gamma & \mathrm{F} \epsilon \\ \mathrm{H} \eta & \mathrm{I} \alpha & \mathrm{A} \gamma & \mathrm{B} \epsilon & \mathrm{C} \zeta & \mathrm{D} \theta & \mathrm{E} \beta & \mathrm{F} \delta & \mathrm{G} \varepsilon \\ \mathrm{I} \theta & \mathrm{A} \beta & \mathrm{B} \delta & \mathrm{C} \varepsilon & \mathrm{D} \eta & \mathrm{E} \alpha & \mathrm{F} \gamma & \mathrm{G} \epsilon & \mathrm{H} \zeta\end{array}$

FIG. 1. A Graeco-Latin square of order 9 with 4 factors.

among the factors.

The statistical model for a Graeco-Latin square is

$$
\begin{gathered}
Y_{i j(k l)=} \mu+\rho_{i}+\gamma_{j}+\beta_{k}+\tau_{l}+\varepsilon_{i j(k l)}, \\
\\
i, j, k, l=1,2, \ldots, n,
\end{gathered}
$$

such that

$$
\sum \rho_{i}=\sum \gamma_{j}=\sum \beta_{k}=\sum \tau_{l}=0,
$$

and the $\varepsilon_{i j(k l)}$ 's are independent and normally distributed with mean zero and variance $\sigma^{2}$.

Thus, for using the Graeco-Latin square design we have to eliminate the interactions. We therefore change the factors to $n, d n, l k$, and $d l$. The problems for the experimental design are as follows.

For given factors $n, d n, l k$, and $d l$, we take $m=n+d n$, then generate randomly, according to a uniform distribution on the interval $[-1,1]$, an $n$ by $m$ matrix $A$; then we generate, according the same distribution, a column and multiply this column by $l k$ and take it as the lower bound $l$; the upper bound $u$ is simply a column obtained by adding $d l$ to each component of $l$.

We suppose the 4 factors are in a range of $(0, M)$, where $M$ is a "big" number, and they satisfy the above statistical model.

We choose 9 levels for each factor, thus $9 \times 9=81$ observations are required. The $9 \times 9$ Graeco-Latin square we used is shown in Figure 1. The corresponding experimental results are given in Table 1. One might have noticed that the running time for cell $(80,45,35,20)$ is 390 seconds while the running time for cell $(100,20,30,30)$ is only 15 seconds. This is because our algorithm employs a line-search sub-algorithm which, sometimes, may require calculating the inverse several times; thus much time is consumed. We note that the rate of success of our algorithm in solving these problems is $100 \%$, i.e. the algorithm always terminates either with a feasible solution or with a detection of inconsistency of the original system. The analysis of variance for the number of iterations is in Table 2; and that for the running time is in Table 3. The runs were performed using MATLAB on a Sun Sparcstation 2.

The hypotheses involving the effects of the 4 factors and the corresponding decisions follow.

- For the number of iterations. (See Table 2)

1. $H_{0_{n}}$ : the effects of all $n$ are equal.

$$
F=\frac{M S_{n}}{M S_{E}} ; \quad F_{8,48,0.95}=2.13 .
$$


TABLE 1

The output of the experiment.
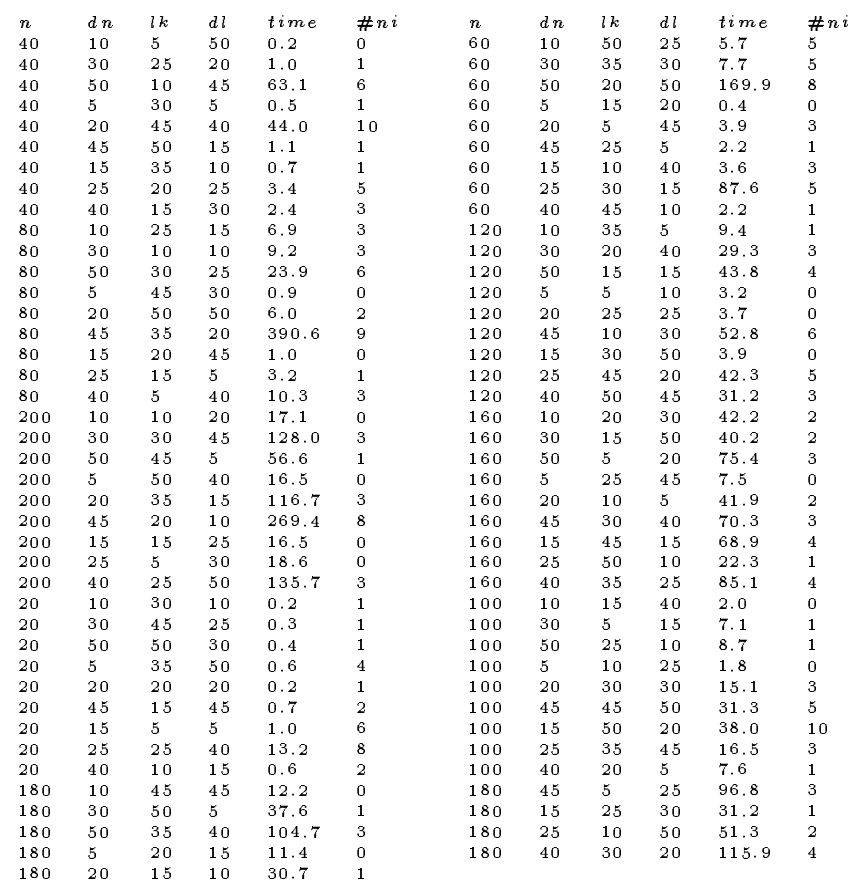

TABLE 2

$A O V$ for the number of iterations.

\begin{tabular}{|l|r|c|c|}
\hline Source of variation & df & SS & MS \\
\hline \hline $\mathrm{n}$ & 8 & 22.9136 & 2.8642 \\
\hline $\mathrm{dn}$ & 8 & 92.6914 & 11.5864 \\
\hline $\mathrm{lk}$ & 8 & 32.2469 & 4.0309 \\
\hline $\mathrm{dl}$ & 8 & 35.5802 & 4.4475 \\
\hline Exp. error & 48 & 309.7037 & 6.4522 \\
\hline Total corrected & 80 & 493.1358 & \\
\hline
\end{tabular}

TABLE 3

AOV for running time(in seconds).

\begin{tabular}{|l|r|c|c|}
\hline Source of variation & df & SS & MS \\
\hline \hline $\mathrm{n}$ & 8 & $5.0336 \times 10^{4}$ & $6.2920 \times 10^{3}$ \\
\hline $\mathrm{dn}$ & 8 & $6.3586 \times 10^{4}$ & $7.9485 \times 10^{3}$ \\
\hline $\mathrm{lk}$ & 8 & $3.5806 \times 10^{4}$ & $4.4758 \times 10^{3}$ \\
\hline $\mathrm{d} \mathrm{l}$ & 8 & $2.2625 \times 10^{4}$ & $2.8281 \times 10^{3}$ \\
\hline Exp. error & 48 & $1.2268 \times 10^{5}$ & $2.5559 \times 10^{3}$ \\
\hline Total corrected & 80 & $2.9504 \times 10^{5}$ & \\
\hline
\end{tabular}




$$
f=\frac{2.8642}{6.4522}=0.4439, \text { so we do not reject } H_{0_{n}} .
$$

2. $H_{0_{d n}}$ : the effects of all $d n$ are equal.

$$
\begin{gathered}
F=\frac{M S_{d n}}{M S_{E}} ; \quad F_{8,48,0.95}=2.13 . \\
f=\frac{11.5864}{6.4522}=1.7957, \text { so we do not reject } H_{0_{d n}} .
\end{gathered}
$$

3. $H_{0_{l k}}$ : the effects of all $l k$ are equal.

$$
\begin{gathered}
F=\frac{M S_{l k}}{M S_{E}} ; \quad F_{8,48,0.95}=2.13 . \\
f=\frac{4.0309}{6.4522}=0.6247, \text { so we do not reject } H_{0 l k} .
\end{gathered}
$$

4. $H_{0_{d l}}$ : the effects of all $d l$ are equal.

$$
\begin{gathered}
F=\frac{M S_{d l}}{M S_{E}} ; \quad F_{8,48,0.95}=2.13 . \\
f=\frac{4.4475}{6.4522}=0.6893, \text { so we do not reject } H_{0_{d l}} .
\end{gathered}
$$

Thus, the number of iterations for solving the feasibility problems is very stable, almost independent of the sizes of problems.

- For the running time. (See Table 3)

1. $H_{0_{n}}$ : the effects of all $n$ are equal.

$$
\begin{gathered}
F=\frac{M S_{n}}{M S_{E}} ; \quad F_{8,48,0.95}=2.13 . \\
f=\frac{6.2920 \times 10^{3}}{2.5559 \times 10^{3}}=2.4618, \text { so we reject } H_{0_{n}} .
\end{gathered}
$$

2. $H_{0_{d n}}$ : the effects of all $d n$ are equal.

$$
\begin{gathered}
F=\frac{M S_{d n}}{M S_{E}} ; \quad F_{8,48,0.95}=2.13 . \\
f=\frac{7.9483^{3}}{2.5559 \times 10^{3}}=3.1098, \text { so we reject } H_{0_{d n}} .
\end{gathered}
$$

3. $H_{0_{l k}}$ : the effects of all $l k$ are equal.

$$
\begin{gathered}
F=\frac{M S_{l k}}{M S_{E}} ; \quad F_{8,48,0.95}=2.13 . \\
f=\frac{4.4758 \times 10^{3}}{2.5559 \times 10^{3}}=1.7512, \text { so we do not reject } H_{0_{l k}} .
\end{gathered}
$$


4. $H_{0_{d l}}$ : the effects of all $d l$ are equal.

$$
\begin{gathered}
F=\frac{M S_{d l}}{M S_{E}} ; \quad F_{8,48,0.95}=2.13 . \\
f=\frac{2.8281 \times 10^{3}}{2.5559 \times 10^{3}}=1.1065, \text { so we do not reject } H_{0_{d l}} .
\end{gathered}
$$

Thus, the running time needed for solving the feasibility problems only depends on $n$, and $m$, i.e. the size of the problem; it does not depend on the lower and upper bounds so much.

4. Solving linear programming problems via weighted centers. In the previous sections we introduced two kinds of centers. We discuss in this section how to use them to design algorithms for solving linear programming problems.

After defining a center, there are several possible ways to use it to solve linear programming problems. For example, we can cut the current polytope by a hyperplane through the current center and throw away the part that does not contain the optimal solution and repeat this procedure; we thus get a sequence of shrinking polytopes which contain the optimal solution, as does Levin [16] as well as Newman [21]; or push the center towards the optimum by adjusting the appropriate bound as done by Renegar [22]. In section 4.1 and 4.2 we will use the same strategies to develop our algorithms via model I and model II respectively. Since the center is usually a solution to a linear system, one natural way to solve the corresponding linear programming problem is to solve a linear system that combines the primal and dual in $R^{m+n}$ and an inequality linking their objective functions. By duality theory, the solution of this system provides solutions of both the primal and the dual problems. However, from a practical viewpoint there are several disadvantages to this approach. First, the combined system is a higher dimensional problem and thus needs more storage and more computational effort; next, since all solutions are within the hyperplane defined by letting the objectives of the primal and the dual be equal, the feasible region has zero volume, and thus some perturbation is necessary to ensure that the interior is not empty and this makes the the computation rather difficult. In section 4.3 we will propose a pulling technique so that, empirically, solving linear programming problem can be reduced to solving a linear system without the above disadvantages.

4.1. Sliding method via model I. The sliding objective function method was first proposed by Yudin and Nemirovskii [31] and Shor [25]. The idea is to reduce the linear programming problem to a sequence of feasibility problems formed by letting the objective be an extra constraint and decreasing the bound corresponding to the objective function as long as it is possible.

Suppose we are to solve (LP). The sliding algorithm based on model I is stated in the following:

Algorithm 4.1

- Initialization. Use model I to find an approximate center $x_{c}$ of the system:

$$
l \leq A^{T} x \leq u
$$

and let $d$ be the corresponding weights. The corresponding ellipsoid is $E^{0}$. Let $u_{0}^{0}:=c^{T} x_{c}$ and

$$
l_{0}^{0}:=c^{T} x_{c}-\left(f\left(d^{0}\right)\left(c^{T}\left(A D A^{T}\right)^{-1} c\right)^{\frac{1}{2}},\right.
$$


so that $l_{0}^{0}$ is tight for the current ellipsoid $E^{0}$. Set $A \leftarrow(c, A)$, i.e., let $c$ be the 0 -th column, and

$$
l^{0}:=\left(l_{0}^{0}, l^{T}\right)^{T}, u^{0}:=\left(u_{0}^{0}, u^{T}\right)^{T} .
$$

Set $k=0$.

- For $k=0,1, \ldots$, do

Use model I to solve system

$$
l^{k} \leq A^{T} x \leq u^{k} \quad\left(\mathrm{FP}_{k}\right)
$$

and get the (approximate) center $x_{c}^{k}$, weights $d^{k}$, and the current ellipsoid $E^{k}$. Let

$$
\begin{gathered}
u_{0}^{k+1}:=c^{T} x_{c}^{k}, \\
l_{0}^{k+1}:=c^{T} x_{c}^{k}-\left(f\left(d^{k}\right)\left(c^{T}\left(A D^{k} A^{T}\right)^{-1} c\right)^{\frac{1}{2}}\right.
\end{gathered}
$$

and $u^{k+1}:=\left(u_{0}^{k+1}, u^{T}\right)^{T} ; l^{k+1}:=\left(l_{0}^{k+1}, l^{T}\right)^{T}$. Set $k \leftarrow k+1$, and repeat.

In the following we analyze its convergence properties. We suppose that the exact center is obtained for each iteration.

THEOREM 4.1. Suppose $\left\{E^{k}\right\}$ is the sequence of ellipsoids generated by the algorithm assuming exact centers are computed. Then we have

$$
\frac{\operatorname{volume}\left(E^{k+1}\right)}{\operatorname{volume}\left(E^{k}\right)} \leq \exp \left(-\frac{1}{2 n}\right)
$$

Proof. Since the bounds $l_{0}^{k}$ and $u_{0}^{k}$ are tight regarding to the current ellipsoid $E^{k}$, by the results in Todd [29], we have

$$
\frac{\operatorname{volume}\left(\tilde{E}^{k+1}\right)}{\operatorname{volume}\left(E^{k}\right)}=\left(\frac{n-1}{n+1}\right)^{\frac{1}{2}}\left(\frac{n^{2}}{n^{2}-1}\right)^{\frac{n}{2}}
$$

where $\tilde{E}^{k+1}$ is the minimum volume ellipsoid that contains

$$
E^{k} \cap\left\{x \in R^{n}: l_{0}^{k} \leq c^{T} x \leq u_{0}^{k}\right\}
$$

(corresponding to $\alpha=0, \beta=1$ in [29]). Since $\tilde{E}^{k+1}$ is a special case of the ellipsoids that we are concerned with, therefore,

$$
\frac{\operatorname{volume}\left(E^{k+1}\right)}{\operatorname{volume}\left(E^{k}\right)} \leq \frac{\operatorname{volume}\left(\tilde{E}^{k+1}\right)}{\operatorname{volume}\left(E^{k}\right)}=\left(\frac{n-1}{n+1}\right)^{\frac{1}{2}}\left(\frac{n^{2}}{n^{2}-1}\right)^{\frac{n}{2}} \leq \exp \left(-\frac{1}{2 n}\right)
$$

with the last inequality following by, e.g., Corollary 3.7.3 of Akgül [1]. $\square$

The above theorem gives us the convergence rate of the algorithm measured by volume of corresponding ellipsoids. As for the convergence rate measured by the objective value, we have, similar to Theorem 6.1 in Goldfarb and Todd [10], 
THEOREM 4.2. Suppose there is a ball $\mathcal{B}$ with a radius of $\delta_{0}$ contained in the feasible region $P$ of $(L P)$. Let $z_{0}:=\max \left\{c^{T} x: x \in \mathcal{B}\right\}, z^{*}:=c^{T} x^{*}$ and $v_{0}=$ $f\left(d^{0}\right) h\left(d^{0}\right)$. Then,

$$
u_{0}^{k}-z^{*} \leq\left(z_{0}-z^{*}\right) \frac{v_{0}^{\frac{1}{2}}}{\delta_{0}} \cdot \exp \left(-\frac{k}{2 n^{2}}\right)
$$

for $k \geq 2 n^{2} \log \left(\delta_{0}^{-1} v_{0}^{\frac{1}{2}}\right)$.

Proof. Let $x^{*}$ be one solution of (LP). Since $P$ contains ball $\mathcal{B}$ it also contains the cone with vertex $x^{*}$ and this ball as its base. Thus, for any $\alpha \in(0,1], \mathcal{B}(\alpha):=$ $\left\{x^{*}+\alpha\left(x-x^{*}\right): x \in \mathcal{B}\right\} \subset P$. The volume of $\mathcal{B}(\alpha)$ is $\alpha^{n} \delta_{0}^{n} \kappa_{n}$ where $\kappa_{n}$ is the volume of the unit ball in $R^{n}$. Denote $T^{k}:=P \cap\left\{x: c^{T} x \geq c^{T} x_{c}^{k}\right\}$, then as long as

$$
T^{k} \cap \mathcal{B}(\alpha) \neq \emptyset
$$

we have

$$
c^{T} x_{c}^{k} \leq \max \left\{c^{T} x: x \in \mathcal{B}(\alpha)\right\}=z^{*}(1-\alpha)+\alpha z_{0},
$$

or,

$$
u_{0}^{k}-z^{*} \leq \alpha\left(z_{0}-z^{*}\right)
$$

We note that if volume $\left(E^{k}\right) \leq \operatorname{volume}(\mathcal{B}(\alpha))$, i.e., $\alpha \geq \frac{v_{0}^{\frac{1}{2}}}{\delta_{0}} \exp \left(-\frac{k}{2 n^{2}}\right)$, (22) holds. Therefore,

$$
u_{0}^{k}-z^{*} \leq\left(z_{0}-z^{*}\right) \frac{v_{0}^{\frac{1}{2}}}{\delta_{0}} \cdot \exp \left(-\frac{k}{2 n^{2}}\right)
$$

Of course, to let the result hold $\alpha$ should be less than or equal to 1 ; this can be ensured by letting $k \geq 2 n^{2} \log \left(\delta_{0}^{-1} v_{0}^{\frac{1}{2}}\right)$.

Thus, this algorithm is a polynomial procedure for solving (LP). Since model I is closely related to the ellipsoid method it can be adapted to a polynomial algorithm for (LP) without getting the exact center, see, e.g., Bland, Goldfarb and Todd [4].

We are now going to discuss a practical stopping criterion. The dual of (LP) is:

$$
\begin{aligned}
& \max l^{T} y_{1}-u^{T} y_{2} \\
& A y_{1}-A y_{2}=c, \\
& y_{1}, y_{2} \geq 0
\end{aligned}
$$

By the results of Burrell and Todd [5], (DP) is equivalent to

$$
\begin{gathered}
\max \psi(y):=l^{T} y_{+}-u^{T} y_{-} \\
A y=c
\end{gathered}
$$

where $y_{-}:=\left(\max \left\{0,-y_{i}\right\}\right), y_{+}:=\left(\max \left\{0, y_{i}\right\}\right)$, and so $y=y_{+}-y_{-}$. By taking

$$
y=\left(c^{T}\left(A D A^{T}\right)^{-1} c\right)^{\frac{1}{2}} D\left(A^{T} z-r\right)
$$

where $z=x_{c}-\left(c^{T}\left(A D A^{T}\right)^{-1} c\right)^{-\frac{1}{2}}\left(A D A^{T}\right)^{-1} c$ and $d$ is such that $f(d)=1$, Burrell and Todd [5] show that

$$
\psi(y) \geq c^{T} z=c^{T} x_{c}-\left(c^{T}\left(A D A^{T}\right)^{-1} c\right)^{\frac{1}{2}} .
$$


We can use $\psi(y)$ as a lower bound on $c^{T} x$. Thus the duality gap associated with $x_{c}^{k}$ and $y$ is:

$$
g a p\left(x_{c}^{k}, y\right)=c^{T} x_{c}^{k}-\psi(y) \leq\left(c^{T}\left(A D A^{T}\right)^{-1} c\right)^{\frac{1}{2}} .
$$

We note that $\left(c^{T}\left(A D A^{T}\right)^{-1} c\right)^{\frac{1}{2}}$, or equivalently, $\left(f(d) c^{T}\left(A D A^{T}\right)^{-1} c\right)^{\frac{1}{2}}$, is half the width of the current ellipsoid along direction $c$. The above theorems ensure that this quantity goes to zero as the ellipsoids shrink. We thus can use it, or equivalently, $f(d) c^{T}\left(A D A^{T}\right)^{-1} c$, as the stopping criterion.

4.2. Obtaining an $\varepsilon$-solution via model II. In this section we describe how to get an $\varepsilon$-solution via model II. Here we define the $\varepsilon$-solution $x^{\varepsilon}$ as a feasible solution with $c^{T} x^{\varepsilon}-z^{*} \leq \varepsilon$. For convenience, we suppose that $\left\|a_{i}\right\|=1$, for all $i=1,2, \ldots, m$, and $\|c\|=1$. The idea is that we use the sliding objective function method via model II and show that if the feasible region of the expanded system (with the objective as its 0 -th constraint) contains a ball $\mathcal{B}$ with radius $\varepsilon$, then the sliding method converges linearly. Thus within finitely many iterations an $\varepsilon$-solution can be obtained. In the following we first state the algorithm, then prove its convergence results and finally, describe some relaxation versions. For convenience, we use $F$ in the following to indicate $F^{I I}$ and $H$ is its Hessian.

\section{Algorithm 4.2}

- Initialization. Use model II to find the (approximate) center $x_{c}$ of the system:

$$
l \leq A^{T} x \leq u
$$

and let $d$ be the corresponding weights. The corresponding ellipsoid is $E^{0}$. Let $u_{0}^{0}:=c^{T} x_{c}$ and

$$
\begin{aligned}
l_{0}^{-1} & :=c^{T} x_{c}-\left(f\left(d^{0}\right)\left(c^{T}\left(A D A^{T}\right)^{-1} c\right)^{\frac{1}{2}}\right. \\
l_{0}^{0} & :=l_{0}^{-1}-q
\end{aligned}
$$

where $q$ is some positive number. Set $A \leftarrow(c, A)$, i.e., let $c$ be the 0 -th column, and

$$
l^{0}:=\left(l_{0}^{0}, l^{T}\right)^{T}, u^{0}:=\left(u_{0}^{0}, u^{T}\right)^{T}
$$

Set $k=0$.

- For $k=0,1, \ldots$, do

(1) Use Algorithm 3.3 to solve system

$$
l^{k} \leq A^{T} x \leq u^{k},
$$

i.e., find $d^{k}=\arg \min \left\{F^{k}(d): d \in R_{+}^{m+1}\right\}$, where the quantities with superscripts are those corresponding to $\left(\mathrm{FP}_{k}\right)$. So $F^{k}(d)$ denotes $F^{I I}(d)$ using $l^{k}$ and $u^{k}$.

(2) Let $x_{c}^{k}$ be the center of the current ellipsoid $E^{k}\left(=E^{I I}\left(d^{k}\right)\right.$ using $l^{k}$ and $u^{k}$ ) and set

$$
u_{0}^{k+1}:=c^{T} x_{c}^{k} \text { and } u^{k+1}:=\left(u_{0}^{k+1}, u^{T}\right)^{T} ;
$$

and $l^{k+1}:=\left(l_{0}^{0}, l^{T}\right)^{T}$. Set $k \leftarrow k+1$, and repeat. 
Note that after setting the lower bound for the 0-th constraint at the initialization step the lower bound for system $\left(\mathrm{FP}_{k}\right)$ will remain the same, and the distance between the optimal value and the 0 -th lower bound is at least $q$.

In the following we discuss the convergence properties of this algorithm. We assume that there is a ball, say $\mathcal{B}$, with radius $\lambda$ contained in $P^{k}$, the feasible region of system $\left(\mathrm{FP}_{k}\right)$. We first prove some lemmas about the sub-iterations in step 1 in the algorithm.

Lemma 4.3. Suppose there is a ball $\mathcal{B}$ with radius $\delta_{0}$ contained in $P^{k}$, and let

$$
\operatorname{Lev}_{k}:=\left\{d \in R_{+}^{m+1}: F^{k}(d) \leq F^{k}(e)\right\}
$$

be the level set. Then, for any $d \in \operatorname{Lev}_{k}$,

$$
\underline{d}^{k}:=\frac{1}{F_{0}^{k}} \leq d_{i} \leq \bar{d}^{k}:=\frac{n F_{0}^{k}}{\delta_{0}^{2}}, \forall i=0,1, \ldots, m
$$

where $F_{0}^{k}:=F^{k}(e)=\left(r^{k}\right)^{T} A^{T}\left(A A^{T}\right)^{-1} A r^{k}-\left(l^{k}\right)^{T} u^{k}+(m+1)$.

Proof. Since $B(d) \leq F^{k}(d) \leq F_{0}^{k}$ for all $d \in \operatorname{Lev}_{k}$, thus, $\frac{1}{d_{i}} \leq F_{0}^{k}$ for all $i$, which leads to the first inequality of (24). As for the second inequality we note that $2 \sqrt{f^{k}(d) a_{i}^{T}\left(A D A^{T}\right)^{-1} a_{i}}$ is the width of ellipsoid $E(d)$ (not $E^{I I}(d)$ ) along $a_{i}$, so

$$
\sqrt{f^{k}(d) a_{i}^{T}\left(A D A^{T}\right)^{-1} a_{i}} \geq \delta_{0} .
$$

Thus,

$$
f^{k}(d) a_{i}^{T}\left(A D A^{T}\right)^{-1} a_{i} \geq \delta_{0}^{2} .
$$

From $\sum_{i=0}^{m} d_{i} a_{i}^{T}\left(A D A^{T}\right)^{-1} a_{i}=n$ and (25), we have, for any $i$,

$$
d_{i} \leq \frac{n}{a_{i}^{T}\left(A D A^{T}\right)^{-1} a_{i}} \leq \frac{n f^{k}(d)}{\delta_{0}^{2}} \leq \frac{n F_{0}^{k}}{\delta_{0}^{2}} .
$$

Thus the proof is complete. $\square$

Without the assumption of $\left(\mathrm{FP}_{k}\right)$ being consistent, the level set might be unbounded. This lemma provides us bounds for the level set in term of the inscribing ball $\mathcal{B}$. The bounds $\underline{d}^{k}$ and $\bar{d}^{k}$ will play important roles in the convergence analysis below.

Lemma 4.4. Under the same assumptions as in Lemma 4.3, if there is $d \in \operatorname{Lev}_{k}$ with

$$
\left\|\nabla F^{k}(d)\right\| \leq \frac{1}{\left(\bar{d}^{k}\right)^{2}}
$$

then $x_{c}(d)$ is feasible for $\left(\mathrm{FP}_{k}\right)$.

Proof. Note that $\nabla F_{i}=-\left(a_{i}^{T} x_{c}(d)-l_{i}^{k}\right)\left(a_{i}^{T} x_{c}(d)-u_{i}^{k}\right)-\frac{1}{d_{i}^{2}}$, for $i=0,1, \ldots, m$. Now (26) implies $\left|\nabla F_{i}\right| \leq \frac{1}{\left(d^{k}\right)^{2}}$. Therefore,

$$
-\left(a_{i}^{T} x_{c}(d)-l_{i}^{k}\right)\left(a_{i}^{T} x_{c}(d)-u_{i}^{k}\right)-\frac{1}{d_{i}^{2}} \geq-\frac{1}{\left(\bar{d}^{k}\right)^{2}}
$$


where

$$
-\left(a_{i}^{T} x_{c}(d)-l_{i}^{k}\right)\left(a_{i}^{T} x_{c}(d)-u_{i}^{k}\right) \geq \frac{1}{d_{i}^{2}}-\frac{1}{\left(\bar{d}^{k}\right)^{2}} \geq 0,
$$

which in turn shows that

$$
l_{i}^{k} \leq a_{i}^{T} x_{c}(d) \leq u_{i}^{k}, i=0,1, \ldots, m,
$$

and thus $x_{c}(d)$ is feasible for $\left(\mathrm{FP}_{k}\right)$.

Now we show that $F^{k}\left(d^{k}\right)$ converges to zero linearly if there is a ball $\mathcal{B}$ with radius $\delta_{\infty}>0$ contained in $P^{k}$ for all $k$. Let

$$
\underline{d}^{\infty}:=\frac{1}{F_{0}^{\infty}}, \bar{d}^{\infty}:=\frac{n F_{0}^{\infty}}{\delta_{\infty}^{2}}
$$

where

$$
F_{0}^{\infty}:=\max \left\{F(e): u_{0} \in\left[z_{0}, u_{0}^{0}\right]\right\}
$$

with $z_{0}:=\max \left\{c^{T} x: x \in \mathcal{B}\right\}$; note that the function $F$ depends on the bounds $l$ and $u$ - here we take the maximum as $u_{0}$ varies. It is obvious that

$$
\underline{d}^{\infty} \leq \underline{d}^{k}, \bar{d}^{\infty} \geq \bar{d}^{k} \text {, for all } k .
$$

Thus, from Lemma 4.3, for all $d \in \operatorname{Lev}_{\infty}:=\cup \operatorname{Lev}_{k}$,

$$
\underline{d}^{\infty} \leq d_{i} \leq \bar{d}^{\infty}, \forall i=0,1, \ldots, m,
$$

and Lemma 4.4 still holds for these bounds. We define

$$
\begin{aligned}
\lambda_{u} & :=\max \left\{\text { largest eigenvalue of } H: u_{0} \in\left[z_{0}, u_{0}^{0}\right], d \in \operatorname{Lev}_{\infty}\right\} \\
\lambda_{l} & :=\min \left\{\text { smallest eigenvalue of } H: u_{0} \in\left[z_{0}, u_{0}^{0}\right], d \in \operatorname{Lev}_{\infty}\right\} .
\end{aligned}
$$

Since $\underline{d}^{\infty}>0, \bar{d}^{\infty}<\infty$ and $\underline{d}^{\infty} \leq d_{i} \leq \bar{d}^{\infty}, \forall i$, both $\lambda_{u}$ and $\lambda_{l}$ are finite and positive.

We now suppose that the output of step 1 of the algorithm $d^{k}$ is such that

$$
\left\|\nabla F^{k}\left(d^{k}\right)\right\| \leq \epsilon \text { with } 0<\epsilon \leq \frac{1}{2\left(d^{\infty}\right)^{2}}<\frac{1}{\left(d^{\infty}\right)^{2}},
$$

$f^{k}\left(d^{k}\right)=B\left(d^{k}\right)$ and $F^{k}\left(d^{k}\right) \leq F^{k}\left(d^{k-1}\right)$ (this holds if we take the initial point as $d^{0}=e$ if $F^{k}(e) \leq F^{k}\left(d^{k-1}\right)$ and $d^{0}=d^{k-1}$ otherwise).

Then from Lemma 4.4, $x_{c}\left(d^{k}\right)$ is feasible to $P^{k}$ and for each $i$

$$
\left(a_{i}^{T} x_{c}-l_{i}\right)\left(a_{i}^{T} x_{c}-u_{i}\right) \in\left(-\frac{1}{2\left(\bar{d}^{\infty}\right)^{2}}-\frac{1}{d_{i}^{2}}, \frac{1}{2\left(\bar{d}^{\infty}\right)^{2}}-\frac{1}{d_{i}^{2}}\right) .
$$

THEOREM 4.5. If we choose $q \geq \frac{3 n^{2}\left(u_{0}^{0}-l_{0}^{-1}\right)}{4}$, then there is a positive number $\delta=\delta\left(m, n, \underline{d}^{\infty}, \bar{d}^{\infty}\right)$ with $0<\delta<1$ such that

$$
\frac{f^{k+1}\left(d^{k+1}\right) \cdot B^{k+1}\left(d^{k+1}\right)}{f^{k}\left(d^{k}\right) \cdot B^{k}\left(d^{k}\right)} \leq \delta .
$$


Proof. By (27), we have, for all $d \in \operatorname{Lev}_{\infty}$,

$$
\begin{aligned}
d_{0} \cdot B(d) & =d_{0} \cdot e^{T} d^{-1}=1+\frac{d_{0}}{d_{1}}+\cdots+\frac{d_{0}}{d_{m}} \\
& \leq 1+\frac{m \bar{d}^{\infty}}{\underline{d}^{\infty}}=: \bar{\delta} .
\end{aligned}
$$

On the other hand, if we let $d$ be $d^{k}$,

$$
\begin{aligned}
\frac{1}{2}\left(u_{0}^{k}-c^{T} x_{c}^{k}\right)^{2} d_{0}^{3} c^{T}\left(A D A^{T}\right)^{-1} c & \leq \frac{1}{2} \frac{u_{0}^{k}-c^{T} x_{c}^{k}}{c^{T} x_{c}^{k}-l_{0}^{k}}\left(\frac{1}{2\left(\bar{d}^{\infty}\right)^{2}}+\frac{1}{d_{0}^{2}}\right) d_{0}^{3} c^{T}\left(A D A^{T}\right)^{-1} c \\
& (\operatorname{using}(29)) \\
\leq & \frac{n}{2} \frac{u_{0}^{k}-c^{T} x_{c}^{k}}{c^{T} x_{c}^{k}-l_{0}^{k}}\left(\frac{d_{0}^{2}}{2\left(\bar{d}^{\infty}\right)^{2}}+1\right) \\
& \left(\text { using } d_{0} c^{T}\left(A D A^{T}\right)^{-1} c \leq n\right) \\
\leq & \frac{3 n}{4} \frac{u_{0}^{k}-c^{T} x_{c}^{k}}{c^{T} x_{c}^{k}-l_{0}^{k}} \\
& \leq \frac{3 n}{4} \frac{u_{0}^{0}-l_{0}^{-1}}{q} \leq \frac{1}{n} .
\end{aligned}
$$

Thus, letting $\Delta u:=u_{0}^{k}-c^{T} x_{c}^{k}$ and $f^{k+1}$ be the function $f$ with $u=u^{k+1}=u^{k}-\Delta u \cdot e_{0}$,

$$
\begin{aligned}
f^{k+1}(d)= & \left(r^{k}\right)^{T} D A^{T}\left(A D A^{T}\right)^{-1} A D r^{k}-\left(l^{k}\right)^{T} D u^{k}-\Delta u d_{0} c^{T} x_{c}^{k} \\
& +\Delta u d_{0} l_{0}^{k}+\frac{1}{4}(\Delta u)^{2} d_{0}^{2} c^{T}\left(A D A^{T}\right)^{-1} c \\
= & f^{k}(d)+d_{0} \Delta u\left(l_{0}^{k}-c^{T} x_{c}^{k}\right)+\frac{1}{4}(\Delta u)^{2} d_{0}^{2} c^{T}\left(A D A^{T}\right)^{-1} c \\
= & f^{k}(d)+d_{0}\left(u_{0}^{k}-c^{T} x_{c}^{k}\right)\left(l_{0}^{k}-c^{T} x_{c}^{k}\right)+\frac{1}{4}(\Delta u)^{2} d_{0}^{2} c^{T}\left(A D A^{T}\right)^{-1} c \\
\leq & f^{k}(d)-\frac{1}{d_{0}}\left(1-\frac{d_{0}^{2}}{2\left(\bar{d}^{\infty}\right)^{2}}\right)+\frac{1}{4}\left(u_{0}^{k}-c^{T} x_{c}^{k}\right)^{2} d_{0}^{2} c^{T}\left(A D A^{T}\right)^{-1} c(\text { using (29)) } \\
\leq & f^{k}(d)-\frac{1}{2 d_{0}}\left(1-\frac{1}{2}\left(u_{0}^{k}-c^{T} x_{c}^{k}\right)^{2} d_{0}^{3} c^{T}\left(A D A^{T}\right)^{-1} c\right) \\
\leq & f^{k}(d)-\frac{1}{2 d_{0}}\left(1-\frac{1}{n}\right) .
\end{aligned}
$$

Suppose the $(k+1)$-st output of step 1 is $d^{k+1}$. Then,

$$
\begin{aligned}
2 \sqrt{\left(f^{k+1}\left(d^{k+1}\right) B\left(d^{k+1}\right)\right.}=f^{k+1}\left(d^{k+1}\right)+B\left(d^{k+1}\right) & \leq f^{k+1}(d)+B(d) \\
& \leq f^{k}(d)+B(d)-\frac{1}{2 d_{0}}\left(1-\frac{1}{n}\right) \\
& =2 \sqrt{f^{k}(d) B(d)}-\frac{1}{2 d_{0}}\left(1-\frac{1}{n}\right) .
\end{aligned}
$$

Thus

$$
\frac{f^{k+1}\left(d^{k+1}\right) B\left(d^{k+1}\right)}{f^{k}(d) B(d)} \leq\left(1-\frac{n-1}{4 n} \frac{1}{d_{0} B(d)}\right)^{2} \leq\left(1-\frac{n-1}{4 n \bar{\delta}}\right)^{2} .
$$


By letting $\delta:=\left(1-\frac{n-1}{4 n \delta}\right)^{2}$ the theorem thus follows.

In the following we consider how to use model II to get a $d^{k}$ satisfying (28) in step 1. For this purpose we let $k^{\prime}$ denote the iteration index within step 1.

Lemma 4.6. Suppose $d^{*}$ is the optimal solution of model II for $F^{k}$. Then for all $d \in \operatorname{Lev}_{\infty}$,

$$
\frac{\lambda_{l}}{2}\left\|d-d^{*}\right\|^{2} \leq F^{k}(d)-F^{k}\left(d^{*}\right) \leq \frac{\lambda_{u}}{2}\left\|d-d^{*}\right\|^{2} .
$$

Proof. It is easy to prove using Taylor's theorem. $\square$

From (19), we have, where $F$ denotes $F^{k}$,

$$
F\left(d^{k^{\prime}+1}\right) \leq F\left(d^{k^{\prime}}\right)-\sigma\left\|\nabla F\left(d^{k^{\prime}}\right)\right\|^{2}
$$

where $\sigma:=\frac{1}{2} \lambda_{u}^{-3} \lambda_{l}^{2}$. From the above result, after at most $\frac{F\left(d^{0}\right)}{\sigma \epsilon}-1$ iterations we have $\left\|\nabla F\left(d^{k^{\prime}}\right)\right\| \leq \epsilon$.

Therefore, for getting a point satisfying (28) in step 1, we only need to take

$$
\epsilon=\frac{1}{2\left(\bar{d}^{\infty}\right)^{2}}
$$

and after using Algorithm 3.3 to get a $d$ with $\|\nabla F(d)\| \leq \epsilon$ (this can be done in finitely many iterations as shown above). The above results also give us a relaxed version of Algorithm 4.2 (we suppose that both $F_{0}^{\infty}$ and $\delta_{\infty}$ are known):

Algorithm 4.3

- Initialization. Use model II to find approximately the center $x_{c}^{0}$ of the system:

$$
l \leq A^{T} x \leq u
$$

and let $d^{0}$ be the corresponding weights. The corresponding ellipsoid is $E^{0}$. Let $u_{0}^{0}:=c^{T} x_{c}^{0}$ and

$$
\begin{aligned}
l_{0}^{-1} & :=c^{T} x_{c}^{0}-\left(f\left(c^{T}\left(A D^{0} A^{T}\right)^{-1} c\right)^{\frac{1}{2}},\right. \\
l_{0}^{0} & :=l_{0}^{-1}-q,
\end{aligned}
$$

where $q \geq \frac{3 n^{2}\left(u_{0}^{0}-l_{0}^{-1}\right)}{4}$. Set $A \leftarrow(c, A)$, i.e., let $c$ be the 0 -th column, and

$$
l^{0}:=\left(l_{0}^{0}, l^{T}\right)^{T}, u^{0}:=\left(u_{0}^{0}, u^{T}\right)^{T} .
$$

Set $k=0$.

- For $k=0,1, \ldots$, do

(1) Use Algorithm 3.3 to solve, approximately, the system

$$
l^{k} \leq A^{T} x \leq u^{k}, \quad\left(\mathrm{FP}_{k}\right)
$$

i.e., find $d^{k}$ such that $\left\|\nabla F^{k}\left(d^{k}\right)\right\| \leq \epsilon$ with $\epsilon$ defined by (30), and scale $d^{k}$ so that $f\left(d^{k}\right)=B\left(d^{k}\right)$.

(2) Let $x_{c}^{k}$ be the center of the current ellipsoid $E^{k}\left(:=E^{I I}\left(d^{k}\right)\right.$ using $l^{k}$ and $u^{k}$ ) and set

$$
u_{0}^{k+1}:=c^{T} x_{c}^{k} \text { and } u^{k+1}:=\left(u_{0}^{k+1}, u^{T}\right)^{T} ;
$$

and $l^{k+1}:=\left(l_{0}^{0}, l^{T}\right)^{T}$. Set $k \leftarrow k+1$, and repeat. 
THEOREM 4.7. Under assumption (A1), Algorithm 4.3 provides an $\varepsilon$-solution of (LP) in finitely many iterations.

Proof. Suppose $\left\{x_{c}^{k}\right\}$ is a sequence of centers generated by Algorithm 4.3 and suppose that for all $k=0,1, \ldots$,

$$
\left|c^{T} x_{c}^{k}-c^{T} x^{*}\right|>\varepsilon .
$$

Then, there is a ball $\mathcal{B}$ with some positive number, say $\delta_{\infty}$, as its radius contained in $P^{k}$ for all $k$. From Theorem $4.5, \frac{v^{k+1}}{v^{k}} \leq \delta$, i.e.,

$$
\frac{\operatorname{volume}\left(E^{k+1}\right)}{\operatorname{volume}\left(E^{k}\right)} \leq \delta^{\frac{n}{2}}
$$

for some $\delta<1$. Since $\mathcal{B} \subset P^{k} \subset E^{k}$ for all $k$, we thus have

$$
0<\kappa_{n} \cdot \delta_{\infty}^{n}=\operatorname{volume}(\mathcal{B}) \leq \operatorname{volume}\left(E^{k}\right) \longrightarrow 0
$$

as $k \longrightarrow \infty$ which is a contradiction. Thus, noting that the number of sub-iterations within step 1 is finite by the argument in the paragraph before Algorithm 4.3, Algorithm 4.3 provides an $\varepsilon$-solution of (LP) in finitely many iterations. $\mathrm{\square}$

The above results can be summarized as follows: if the algorithm does not converge then a linear convergence rate for the volume of the corresponding ellipsoid can be proved; thus the algorithms do converge and the rate of shrinking of the volume of the ellipsoid is reduced as $x_{c}^{k}$ approaches $x^{*}$. It can be shown, by Lemma 4.6, that if $F^{k} \longrightarrow 0$ linearly, $x_{c}^{k} \longrightarrow x^{*}$ linearly. The linear convergence rate is most likely the best. An extreme example is when $m+1=n$ and after putting in the objective vector, the system becomes an $n \times n$ square system; thus $x_{c}^{k}=A^{-1} r^{k}$ and $x_{c}^{k} \longrightarrow x^{*}$ at most linearly.

4.3. Computational techniques and numerical results. In this section, we discuss implementation techniques when using models I and II and report preliminary numerical results with Algorithm 4.2 when applied to problem (LP).

When we solve (LP), model I or model II is used recursively. Therefore the techniques in section 3.5 apply here.

For given $d \in R_{+}^{m+1}$, consider $x_{c}(d)$ as a function of $l_{0}$, the lower bound imposed on the objective function $c^{T} x$. Then we have

$$
c^{T} x_{c}(d) \longrightarrow-\infty \text { as } l_{0} \longrightarrow-\infty .
$$

This suggests that if $l_{0}$ is chosen to be a very large negative number, finding an approximate solution of (LP) might need only one iteration, i.e., be reduced to a feasibility problem (FP). We call this technique the "pulling" technique, since it pulls the current center to the optimal solution. Note that most "bound-update" methods, e.g., Levin [16], Newman [21] and Renegar [22], decrease an appropriate bound so as to "push" the current center to approach the optimal solution. Our limited test results are reported in Table 4. The test problems we use are those in Avis and Chvatal [2] but with upper bounds on each component of $x$, namely,

$$
\begin{aligned}
& \max e^{T} x \\
& N x \leq 10^{4} e, \\
& 0 \leq x \leq 10 e .
\end{aligned}
$$


TABLE 4

Computational results of Algorithm 3.3.

\begin{tabular}{|c|c|c|}
\hline Dimension & SMPLX & ALGORITHM 3.3 \\
\hline \hline 10 & \# of iter=12 & \# of iter $=3(9+40+6), \Delta=2.9 \times 10^{-4}$ \\
\hline 20 & \# of iter $=30$ & \# of iter $=3(9+58+5), \Delta=9.1 \times 10^{-4}$ \\
\hline 30 & \# of iter $=37$ & \# of iter $=4(11+71+7+11), \Delta=6.6 \times 10^{-6}$ \\
\hline 40 & \# of iter $=85$ & \# of iter $=3(16+152+12), \Delta=5.4 \times 10^{-4}$ \\
\hline 50 & \# of iter $=128$ & \# of iter $=4(9+39+16+7), \Delta=6.9 \times 10^{-4}$ \\
\hline
\end{tabular}

TABLE 5

The effect of $q$ on iteration number.

\begin{tabular}{|l||l|l|l|}
\hline & dimension=30 & dimension=40 & dimension=50 \\
\hline \hline$q=10$ & $>35$ & $>35$ & $>35$ \\
\hline$q=10^{2}$ & $>35$ & $>35$ & $>35$ \\
\hline$q=10^{3}$ & $>35(>114)$ & $>35(>121)$ & $>35$ \\
\hline$q=10^{4}$ & $27(11+8+4 \cdot 5+21 \cdot 4=87)$ & $18(16+13+2 \cdot 5+7 \cdot 4+7 \cdot 3=88)$ & $>35(>134)$ \\
\hline$q=10^{5}$ & $9(11+16+8+17+5 \cdot 6=82)$ & $6(16+17+13+12+6+6=70)$ & $11(9+16+7+9+2 \cdot 6+5 \cdot 5=102)$ \\
\hline$q=10^{6}$ & $5(11+27+12+7+9=66)$ & $4(16+50+8+7=81)$ & $6(9+20+8+7+8+8=60)$ \\
\hline$q=10^{7}$ & $4(11+43+8+12=74)$ & $3(16+76+12=104)$ & $4(9+38+15+8=70)$ \\
\hline$q=10^{8}$ & $4(11+71+7+11=100)$ & $3(16+152+12=180)$ & $4(9+71+14+11=105)$ \\
\hline
\end{tabular}

$N$ is an $n \times n$ matrix with integer elements chosen randomly in the range $1, \ldots, 1000$. We reduce (32) to our form (LP):

$$
\begin{aligned}
& \max e^{T} x \\
& l \leq \tilde{N} x \leq u
\end{aligned}
$$

with

$$
l=0, \tilde{N}=\left(\begin{array}{c}
N \\
I
\end{array}\right), u=\left(10^{4} e^{T}, 10 e^{T}\right)^{T} .
$$

We choose $q=10^{8}$. The data in the column corresponding to the simplex method are from Nazareth [20]; these are average numbers of iterations for a number of random problems. $\Delta:=f(d) c^{T}\left(A D A^{T}\right)^{-1} c$ is the width of the ellipsoid in the direction $c$ after the final iteration and it serves here as the stopping criterion (we terminate if $\left.\Delta \leq 10^{-3}\right)$. The stopping criterion for model II is $\|\nabla F(d)\| \leq 10^{-5}$. The numbers in the parentheses are the numbers of iterations of Algorithm 3.3 for obtaining each approximate center. Table 5 and Table 6 show the effects of $q$ on the iteration numbers and running times respectively. They also show that the effect of "pulling" is much more significant than that of "pushing". Thus, choosing an appropriate $q$ will save computational effort. Again, the runs were performed using MATLAB on a Sun Sparcstation 2.

5. Summary and conclusions. In this paper we have analyzed, and tested, some generalized models for linear systems and, later, proposed some algorithms for solving linear programming problems via these models. Foremost among these is the idea of generalizing Burrell and Todd's approach [5] which is closely related to the coordinate descent method so that Newton's method can be employed. We develop our models in sections 2 and 3. The key result is that the function $v(d)=f(d) h(d)$ where $v$ is such that $\kappa_{n} v^{\frac{n}{2}}$ is the volume of the ellipsoid can be, equivalently for our purpose, replaced by $F(d)=f(d)+h(d)$, because $f(d)$ and $h(d)$ are homogeneous functions of degrees 1 and -1 respectively. The first model, model $I$, is thus established 
TABLE 6

The effect of $q$ on running time in seconds.

\begin{tabular}{|l||c|c|c|}
\hline & dimension $=30$ & dimension $=40$ & dimension $=50$ \\
\hline \hline$q=10$ & $>57$ & $>93$ & $>177$ \\
\hline$q=10^{2}$ & $>57$ & $>93$ & $>177$ \\
\hline$q=10^{3}$ & $>57$ & $>93$ & $>177$ \\
\hline$q=10^{4}$ & 55 & 65 & $>177$ \\
\hline$q=10^{5}$ & 61 & 74 & 119 \\
\hline$q=10^{6}$ & 48 & 58 & 72 \\
\hline$q=10^{7}$ & 41 & 90 & 108 \\
\hline$q=10^{8}$ & 43 & 146 & 124 \\
\hline
\end{tabular}

by using $F(d)=f(d)+h(d)$ as the objective function. The advantage of this model is that it is a convex program as well as preserving the advantages of Burrell and Todd's approach [5]. We prove the existence and uniqueness of the smallest ellipsoid of the form (3) which contains the feasible region $P$. The center of this smallest ellipsoid is proved to be an interior point of $P$; it is our first kind of center. For solving model I we propose two algorithms: the first one is a coordinate descent method obtained by modifying the Liao and Todd algorithm [18]. It is proved to have a polynomial time bound. Since the objective function of model I might be not strictly convex, it thus prevents the use of a full version of Newton's method. To overcome this disadvantage we replace $h(d)$ by a strictly convex function $B(d)$ which forms our second model, model II. Similarly, we propose two algorithms for solving this model: a coordinate descent algorithm and a Newton's algorithm. The first one is actually a ball-like method [4] which has been shown to be not a polynomial time algorithm. The second algorithm is a combination of Newton's algorithm and a scaling technique, which works well in practice. The statistical analysis shows that this algorithm is very robust numerically. We also provide a rather detailed analysis of the outputs of these models according to their minimal values over their effective domains.

In section 4 we develop methods for solving linear programming problems via these models. They are basically sliding objective function methods. The first one is based on model I. We prove its geometrical convergence rates measured by the volume of the corresponding ellipsoid and by the objective value of the linear program respectively. The second algorithm, based on model II, provides an $\varepsilon$-solution of (LP) in finitely many iterations. One property of this approach is that if $c^{T} x_{c}$, the current objective value, is $\varepsilon$ far away from $z^{*}$, the optimal value, a constant decrease (depending on $\varepsilon)$ can be obtained. Or in other words, if $x_{c}$ does not approach the optimal set, $x_{c}$ approaches the optimal set linearly. In addition to the pushing technique similar to that using by the sliding objective function method [4] and Renegar [22] we also propose a "pulling" technique: we let the lower bound corresponding to the objective be a very large negative number, i.e., $l_{0} \sim-\infty$ (or $u_{0} \sim \infty$ for the maximizing linear program). With the help of this pulling technique, solving (LP) is almost equivalent to solving a feasibility problem as shown by the numerical tests.

Finally, we comment on the major unfinished tasks that we believe are evident from this paper. The biggest one is, as mentioned above, to analyze our approach to see if Newton's algorithm is polynomial for linear programming problems within our framework. We list below some of our suggestions for further work along this direction:

(1). We showed that if $f(d)<0$ for some $d \geq 0$ then the system (FP) is incon- 
sistent. Further research might investigate the number of iterations needed to find the infeasibility if the system is not consistent.

(2). We gave artificial examples showing that, in the case that $\operatorname{int}(P)=\emptyset$ but $P \neq \emptyset$, for both model I and model II, there exist sequences $\left\{x_{c}\left(d^{k}\right)\right\}$ which fail to approach the feasible region although $d^{k}$ is such that $F\left(d^{k}\right)\left(\right.$ or $\left.F^{I I}\left(d^{k}\right)\right)$ approaches zero. But Algorithm 3.3 always gave feasible solutions in our computational tests. Investigation is thus needed for this kind of behavior of the algorithm.

(3). From the numerical test, we find that the "pulling" technique is an important factor with regard to the number of major iterations, i.e. number of applications of model II. The bigger $q$, the fewer major iterations. We thus hope to prove that solving (LP) is equivalent to finding the center of $\left(\mathrm{FP}_{0}\right)$ with a large $q$. 


\section{REFERENCES}

[1] M. AkgüL, Topics in relaxation and ellipsoidal methods, Pitman Advanced Publishing Program, 1984.

[2] D. Avis and V. Chvatal, Notes on Bland's pivoting rule, Mathematical Programming Study, 8 (1978), pp. 24-34.

[3] R. M. Bethea, B. S. Duran, and T. L. Boullion, Statistical Methods, Marcel Dekker, Inc., 1985 .

[4] R. G. Bland, D. Goldfarb, And M. J. Todd, The ellipsoid method: a survey, Operations Research, 29 (1981), pp. 1039-1091.

[5] B. P. Burrell AND M. J. TodD, The ellipsoid method generates dual variables, Mathematics of Operations Research, 10 (1985), pp. 688-700.

[6] V. Chvátal, Linear Programming, W. H. Freeman and Company, 1980.

[7] T. F. Coleman and C. V. Loan, Handbook for Matrix Computations, SIAM, 1988.

[8] J. L. Devore, Probability and Statistics, Brooks/Cole Publishing Company, 1991.

[9] J. L. Goffin, On the non-polynomiality of the relaxation method for system of inequalities, Technical Report, Faculty of Management, McGill University, Montreal, Quebec, 1979.

[10] D. Goldfarb AND M. J. TODD, Modifications and implementation of the ellipsoid algorithm for linear programming, Mathematical Programming, 23 (1982), pp. 1-19.

[11] G. H. Golub and C. V. Loan, Matrix Computations, The Johns Hopkins University Press, Baltimore, Maryland, 1989.

[12] R. A. Horn And C. R. Johnson, Matrix Analysis, Cambridge University Press, Cambridge, 1990.

[13] F. JoHn, Extremum problems with inequalities as subsidiary conditions, in Studies and Essays(Courant Anniversary Volume), Interscience, New York, 1948.

[14] L. G. Khachiyan, A polynomial algorithm for linear programming, Doklady Akad. Nauk. USSR, 244 (1979), pp. 1093-1096.

[15] L. G. KHAChIYAN AND M. J. TODD, On the complexity of approximating the maximal inscribed ellipsoid for a polytope, Technical Report 893, School of Operations Research and Industrial Engineering, Cornell University, 1990.

[16] A. Y. LEVIN, On an algorithm for the minimization of convex functions, Soviet Mathematics Doklady, 6 (1965), pp. 286-290.

[17] A. LiaO, Algorithms for Linear Programming via Weighted Centers, PhD thesis, Cornell University, Ithaca, New York, 1992.

[18] A. LiAO AND M. J. TODD, The ellipsoid algorithm using parallel cuts, Technical Report 997, School of Operations Research and Industrial Engineering, Cornell University, 1992.

[19] C. B. Moler, J. Little, S. Bangret, and S. Kleiman, Pro-Matlab User's Guide, The MathWorks, Inc., 1987.

[20] J. L. Nazareth, Pricing criteria in linear programming, in Progress in Mathematical Programming, N. Meggiddo, ed., New York, 1989, Springer-Verlag, pp. 105-130.

[21] D. J. Newman, Location of the maximum on unimodal surfaces, Journal of the Association for Computing Machinery, 12 (1965), pp. 395-398.

[22] J. RENEGAR, A polynomial-time algorithm based on Newton's method for linear programming, Mathematical Programming, 40 (1988), pp. 59-93.

[23] R. T. Rockafellar, Convex Analysis, Princeton University Press, 1970.

[24] N. Z. SHOR, Utilization of the operation of space dilatation in the minimization of convex functions, Cybernetics, 6 (1970), pp. 7-15.

[25] - Cut-off method with space extension in convex programming problems, Cybernetics, 13 (1977), pp. 94-96.

[26] G. SONNEVEND, An analytical center for polyhedrons and new classes of global algorithms for linear (smooth, convex) programming, in Lecture Notes in Control and Information Sciences, No. 84, Berlin, 1986, Springer-Verlag, pp. 866-875.

[27] S. P. Tarasov, L. G. Khachiyan, and I. I. Erlich, The method of inscribed ellipsoids, Soviet Math. Dokl., 37 (1988).

[28] M. J. TODD, Some remarks on the relaxation method for linear inequalities, Technical Report 419, School of Operations Research and Industrial Engineering, Cornell University, 1979.

[29] — On minimum volume ellipsoids containing part of a given ellipsoid, Mathematics of Operations Research, 7 (1980), pp. 253-261.

[30] P. M. VAIDYA, A new algorithm for minimizing convex functions over convex sets, Technical Report, AT\&T Bell Laboratories, 1989.

[31] D. B. YUdin AND A. S. Nemirovskir, Informational complexity and efficient methods for the solution of convex extremal problems, Matekon, 13 (1976), pp. 3-25. 